\title{
Globular Clusters and X-ray Point Sources in Centaurus A (NGC 5128)
}

\author{
Kristin A. Woodley ${ }^{1}$, Somak Raychaudhury ${ }^{2,3}$, Ralph P. Kraft ${ }^{2}$, William E. Harris ${ }^{1}$, Andrés \\ Jordán $^{2,4}$, Katherine E. Whitaker ${ }^{2,5}$, Christine Jones ${ }^{2}$, William R. Forman ${ }^{2}$, Stephen S. \\ Murray $^{2}$
}

\begin{abstract}
We detect 353 X-ray point sources, mostly low-mass X-ray binaries (LMXBs), in four Chandra observations of Centaurus A (NGC 5128), the nearest giant earlytype galaxy, and correlate this point source population with the largest available ensemble of confirmed and likely globular clusters associated with this galaxy. Of the X-ray sources, 31 are coincident with 30 globular clusters that are confirmed members of the galaxy by radial velocity measurement (2 X-ray sources match one globular cluster within our search radius), while 1 X-ray source coincides with a globular cluster resolved by HST images. Another $36 \mathrm{X}$-ray point sources match probable, but spectroscopically unconfirmed, globular cluster candidates. The color distribution of globular clusters and cluster candidates in Cen A is bimodal, and the probability that a red, metal rich GC candidate contains an LMXB is at least 1.7 times that of a blue, metal poor one. If we consider only spectroscopically confirmed GCs, this ratio increases to $\sim 3$. We find that LMXBs appear preferentially in more luminous (massive) GCs. These two effects are independent, and the latter is likely a consequence of enhanced dynamical encounter rates in more massive clusters which have on average denser cores. The X-ray luminosity functions of the LMXBs found in GCs and of those that
\end{abstract}

\footnotetext{
${ }^{1}$ Department of Physics \& Astronomy, McMaster University, Hamilton ON L8S 4M1, Canada; woodleka@physics.mcmaster.ca, harris@physics.mcmaster.ca

${ }^{2}$ Harvard-Smithsonian Center for Astrophysics, 60 Garden Street, MS-67, Cambridge, MA 02138, USA; kraft@head.cfa.harvard.edu, ajordan@cfa.harvard.edu, cjf@head.cfa.harvard.edu,wrf@head.cfa.harvard.edu, ssm@head.cfa.harvard.edu

${ }^{3}$ School of Physics \& Astronomy, University of Birmingham, Birmingham B15 2TT, UK; somak@star.sr.bham.ac.uk

${ }^{4}$ European Southern Observatory, Karl-Schwarzschild-Str. 285748 Garching bei München, Germany

${ }^{5}$ Department of Astronomy, Yale University, 260 Whitney Avenue, New Haven, CT 06511, USA; katherine.whitaker@yale.edu
} 
are unmatched with GCs reveal similar underlying populations, though there is some indication that fewer X-ray faint LMXBs are found in globular clusters than X-ray bright ones. Our results agree with previous observations of the connection of GCs and LMXBs in early-type galaxies and extend previous work on Centaurus A.

Subject headings: galaxies: elliptical and lenticular - galaxies: individual (NGC 5128) — globular clusters: general - X-rays: galaxies - X-rays: binaries

\section{Introduction}

Observations of early-type galaxies with the Chandra X-ray Observatory have shown that a significant fraction of low-mass X-ray binaries (LMXBs) in these galaxies are associated with globular clusters (GCs) (see Fabbiano 2006, for a review). The fraction of LMXBs identified with known GCs around massive early-type galaxies varies from at least $20 \%$ in NGC 4697 (Sarazin et al. 2001) to a remarkable 70\% in NGC 1399, the central giant elliptical of the Fornax cluster (Angelini et al. 2001). In the Milky Way $\sim 10 \%$ of all bright LMXBs are found in GCs, even though the GCs account for $<10^{-3}$ of the stellar mass of the galaxy (e.g. Katz 1975; Grindlav 1993).

The overabundance of LMXBs in GCs is thought to be due to their high central densities, which result in greatly enhanced rates of dynamical interactions with respect to the field. These interactions can lead to the creation of LMXB progenitors via dissipative encounters between neutron stars and ordinary stars (e.g. Fabian et al. 1975), or encounters in which a compact object replaces a member of a binary in a three-body interaction (e.g. Hills 1976). This picture is supported by observations in the Milky Way and nearby early-type galaxies (Pooley et al. 2003; Jordán et al. 2004; Sivakoff et al. 2007; Jordán et al. 2007) which show that estimates of GC dynamical collision rates are good predictors for the presence of LMXBs in GCs.

It has even been suggested (White et al. 2002) that almost all LMXBs are formed in the cores of GCs and either are later ejected from their host clusters due to the evaporation of the GCs or escape the cluster due to dynamical processes or kick velocities imparted at birth. Recent analyses suggest that this is not the case though, as observations seem to require the presence of a bona fide population of field LMXBs besides a component whose formation is connected to GCs (Irwin 2005; Juett 2005). Moreover, Fabbiano et al. (2007) have recently shown that GCs in NGC 3379 lack low luminosity LMXBs when compared to the field population, implying that the latter cannot have originated from GCs (see also 
Voss \& Gilfanov 2007). To date, large populations of LMXBs have been found in X-ray observations of E/S0 galaxies over a wide range of luminosities (e.g. Sivakoff et al. 2007; Kundu et al. 2007) and in large disk galaxies such as M31, NGC 3115, and NGC 4594 (Fan et al. 2005; Kundu et al. 2003; Di Stefano et al. 2003).

In this paper, we present results from a correlation of the LMXB population, detected as point sources in Chandra/ACIS observations, with the optically detected GC population in the galaxy Centaurus A (Cen A, NGC 5128). A previous study of the connection between GCs and LMXBs in Cen A, using smaller optical and X-ray catalogs, is presented in Minniti et al. (2004). Cen $\mathrm{A}$ is the nearest massive early-type galaxy $\left(M_{B}=-21.1\right)$ (Dufour et al. 1979), and has been widely studied across the electromagnetic spectrum (for a comprehensive review, see Israel 1998). X-ray observations of Cen A (Feigelson et al. 1981; Turner et al. 1997; Kraft et al. 2000, 2002, 2003a,b; Hardcastle et al. 2007) reveal several distinct emission components, including a bright, compact and variable nucleus, an X-ray jet perpendicular to the dust lane and aligned with radio features, and diffuse emission representing the hot phase of the interstellar medium. In addition, several hundred bright point sources are seen in X-rays (Kraft et al. 2001; Voss \& Gilfanov 2006), mostly representing the LMXB population.

Studying Cen A has major advantages over studies of the LMXB/GC connection in other early-type galaxies. First, Cen $\mathrm{A}$ is $\sim 5$ times closer than the often-studied galaxies in the Virgo and Fornax clusters, which allows for a much deeper census and better characterization of its X-ray binary population. Second, at the distance of Cen A, a scale of $1^{\prime \prime}$ corresponds to a linear distance of just $18 \mathrm{pc}$, which is comparable to the physical diameters of GCs. Furthermore, Cen A has a moderately large GC population $(\simeq 1500$ clusters; see Harris et al. 2006), with a bimodal distribution in color, equally split between red and blue.

An intriguing aspect of the observations linking GCs with X-ray point sources has been that LMXBs in early-type galaxies are more likely to be found in the redder (highermetallicity Z) GCs. This trend already can be seen in the small Milky Way sample of LMXBs, but becomes much more evident in larger galaxies (e.g. Angelini et al. 2001; Kundu et al. 2002; Di Stefano et al. 2003; Jordán et al. 2004; Kim et al. 2006; Sivakoff et al. 2007; Posson-Brown et al. 2007; Kundu et al. 2007). The physical mechanisms underlying this observational result are still unknown, although several possibilities have been put forward, including metallicity dependent variations in the initial mass function (Grindlav 1987), the link between metallicity and the outer convective zones of stars (Ivanova 2006) and the effects of metallicity on radiation induced stellar winds (Maccarone et al. 2004).

A recent spectroscopically based survey of the cluster ages $(\sim 150$ GCs with Lick index measurements) by Beasley et al. (2007) indicates that the great majority of the GCs are old, 
with ages $\tau \gtrsim 10$ Gyr. However, perhaps $20 \%$ of them (almost all on the metal-rich side) scatter to younger ages in the 6 to 8 Gyr range. For comparison, Rejkuba et al. (2005) show that the field halo stars in Cen A have a mean inferred age of $8_{-3.5}^{+3}$ Gyr. In the Milky Way, the metal-rich clusters, including the LMXB hosts, are mostly old systems (Rosenberg et al. 1999). The two giant galaxies M87 and M49 in Virgo are found to have mostly old GC systems (Cohen et al 1998; Jordán et al. 2002; Puzia et al 1999; Beasley et al. 2000) and also show a preference for metal-rich GCs to host LMXBs (Sivakoff et al. 2007). Thus the preference of X-ray binaries to be in higher metallicity clusters does not seem to be associated with age and must be primarily due to metallicity (see also Kundu et al. 2003).

Recent work by Jordán et al. (2007) exploits the distance to Cen A in order to explore the detailed dependence of the incidence of LMXBs in GCs on the structural parameters of GCs such as their half-light radii and central densities. Jordán et al. (2007) conclude that neither concentration nor mass are fundamental variables in determining the presence of LMXBs in GCs, and that the more fundamental parameters relate to central density and size.

Various studies indicate that the half-light radii of metal-rich GCs in large E galaxies are $\sim 20 \%$ smaller than the metal-poor clusters (see Kundu \& Whitmore (2001), Jordán et al. (2005), and Gómez \& Woodley (2007) among others). Jordán (2004) shows that this could be a result of mass segregation and the longer lifetimes of lower metallicity stars for a given mass, under the assumption that the half-mass radii distribution does not depend on metallicity. In this case, we expect dynamical effects to be largely decoupled from the metallicity of GCs, but if the half-light radii reflect indeed a difference in half-mass radii at least part of the preference of LMXBs for metal-rich GCs could be due to their smaller average size and therefore denser cores. It is easy to show that dynamical processes cannot be responsible for all of the observed effect of metallicity on the incidence of LMXBs, even if there was a size difference consistent with that observed in other early-type galaxies. Assuming encounter rates scale with half-light radii $r_{h}$ as $r_{h}^{-2.5}$ (see Sivakoff et al. 2007) metal-rich GCs would need to be 50\% smaller than their metal-poor counterparts in order to fully account for a threefold enhancement of the number of LMXBs in metal-rich GCs. This level of size difference is ruled out by the observations. The metallicity itself seems to be an important factor in producing LMXBs.

This paper is organized as follows. We introduce the observations and discuss data reduction and point source detection in \$2, In \$3, we identify GCs from optical observations which are associated with the X-ray point sources, and discuss their properties in 94 . In \$5, we examine the X-ray properties of the point sources that are associated with GCs and compare with those not found in GCs. Finally, results are summarized in $\$ 6$. In this analysis, we adopt 
a distance of $3.8 \pm 0.2 \mathrm{Mpc}$ for Cen A (an average of five precise standard candles including TRGB, PNLF, SBF, LVPs, and Cepheids; see McLaughlin et al. 2007; Ferrarese et al. 2007, and references therein) and an integrated optical luminosity $M_{V}^{t}=-22.0$ (Dufour et al. 1979).

\section{X-ray Observations}

\subsection{Chandra/ACIS data: Preparation and Analysis}

We first present the analysis of four ACIS observations of Centaurus A with the Chandra X-ray Observatory. A summary of the observation log is given in Table 1. All observations were made in FAINT mode. The level 1 events files of all four data sets were reprocessed to remove the pixel randomization, apply CTI correction, and remove flaring and hot pixels. The work described here includes a re-processing/re-analysis of our results presented in Kraft et al. (2001). For every ACIS observation, X-ray light curves were extracted for each $\mathrm{CCD}$ in the 5.0-10.0 keV band, excluding the nucleus and point sources, to search for background flares. All periods when the background exceeded the mean by $3 \sigma$ were removed. Total good times of the ACIS-S and ACIS-I observations are $\sim 94 \mathrm{ks}$ and $\sim 68 \mathrm{ks}$, respectively.

The four data sets were aligned relative to each other by comparing the positions of 30 bright point sources within $3^{\prime}$ of the nucleus. The coordinates of the data sets were adjusted to minimize the root mean square separations of the ensemble of sources. The largest adjustment made to the astrometry for any data set was $1.2^{\prime \prime}$. We also investigated whether any of the data sets were offset in roll angle, but found that no adjustment to the roll was necessary. These four data sets were then aligned on the sky in absolute coordinates by co-aligning the X-ray and radio nuclei. We estimate that the four data sets are aligned relative to each other to better than $0.1^{\prime \prime}$, and in absolute sky coordinates to better than $0.5^{\prime \prime}$.

\subsection{Point Source Detection}

The detection of point sources was performed on five data sets, individually on each of the four observations, and on the combined events file of the 2002 and 2003 ACIS-S observations (OBSID 2978 \& 3965). In general, we found it preferable to perform point source detection individually on each of the four data sets. Any improvements in signal to noise by combining all four data sets would be more than offset by the complexity introduced by the process. However, since the AO-3 and AO-4 observations were made with the same 
detector (ACIS-S) and with nearly identical roll angles, we have used the combination of these two observations.

For each of the five data sets, we created images at full resolution (i.e. 1 pixel $\equiv$ $\left.0.492^{\prime \prime}\right)$ in three energy bands for each CCD, namely soft $(0.5-2.0 \mathrm{keV})$, hard $(2.0-5.0 \mathrm{keV})$ and combined (0.5-5.0 keV) bands. In all cases, the background far exceeds the nominal ACIS particle background over much of the FOV. The background in the soft band images is dominated by emission from hot gas in Cen A. The morphology of this emission has a complex spatial dependence, and the sensitivity is also spatially modulated by the variable absorption of the dust lane. The background in the hard band is dominated by the wings of the point spread function (PSF) of the bright active nucleus. This is most significant within $\sim 1^{\prime}$ of the nucleus, but the X-ray AGN is so bright that it adds significantly to the background over the entire ACIS FOV.

Point source detection was performed with the CIAO software package wavdetect on spatial scales of 1.0, 1.414, 2.0, 2.828, 4.0, 5.656, 8.0, 11.312, 16.0 pixels, with a threshold of $10^{-6}$. Source detection was performed separately on each chip. We expect to detect roughly one false source per CCD at the sensitivity limit. We detect 353 sources, of which $\sim 10$ are statistically likely to be mis-identified fluctuations in the background. The spatial distribution of sources is strongly peaked toward the center of the galaxy, demonstrating that the vast majority lie within Cen A and not unrelated foreground or background objects. The limiting sensitivity of these observations, however, is a widely non-uniform function of position on the sky, due to the spatial distribution of the ISM, differential absorption of the dust lane, the scatter from the nucleus, and the complex spatial dependence of the telescope PSF. Point sources detected in the two ACIS-I observations are complete and unbiased (defined as a $4 \sigma$ measurement of the luminosity) to a luminosity of $2 \times 10^{37} \mathrm{ergs} \mathrm{s}^{-1}$ in the 0.1-10.0 keV band (unabsorbed). The limiting sensitivity of the two ACIS-S observations is roughly a factor of two lower than this because of the longer exposure, greater sensitivity of the backside illuminated CCD, and the position of the telescope best focus at the nucleus.

We performed a count rate to luminosity conversion assuming a $5 \mathrm{keV}$ bremsstrahlung spectrum, typical of Galactic X-ray binaries, and absorption $\left(N_{H}=8.0 \times 10^{20} \mathrm{~cm}^{-2}\right)$ by foreground gas in our galaxy. The conversions for each CCD in each AO were computed with the CXC program PIMMS. All count rates were exposure corrected and background subtracted. The count rate was estimated from the broad band $(0.5-5.0 \mathrm{keV})$ image, even if the source was detected in only one or two of the bands. The source background in each band was determined using the residual background image from wavdetect. We also fit bremsstrahlung and power law models to all sources with more than 50 counts in any observation. With a few exceptions of heavily absorbed sources, the luminosities derived by the two methods are 
in general statistically consistent.

We will describe the spectral properties of the ensemble of sources in detail in a future publication. The photometric properties of a subset of these point sources, based on some or all of these observations, have been discussed elsewhere by Kraft et al. (2001) and Voss \& Gilfanov (2006). For the remainder of this paper, we concentrate on describing the identification of the X-ray point sources with the GCs in the galaxy and the optical and X-ray properties of the matched GC-LMXB pairs.

\section{X-ray Point Sources in Globular Clusters}

In the three decades since the breakthrough discovery of just one of its GCs by Graham \& Phillips (1980), 415 GCs have been found in Cen A, through either radial velocity measurement or high resolution imaging. Cen A, with a low Galactic latitude of $b=19^{\circ}$, is in a field contaminated by a large number of foreground stars. There are also a large number of more distant galaxies in the field, since our target is in the foreground of the Hydra-Centaurus and Shapley superclusters (Raychaudhurv 1989). Many contaminant stars and galaxies have colors similar to those of GCs (e.g. Harris, Harris \& Geisler 2004). While the use of colors, morphologies, and sizes of objects all help to reduce the contamination from the field and select GC candidates, none are definitive methods for classification. Radial velocity measurements and high-resolution imaging (particularly with $H S T$ ) are the best ways to confirm an optically-identified object as a definite GC in this challenging galaxy.

In this analysis, we use the complete catalog of 415 identified GCs published in Woodley et al. (2007). Of these, 340 have radial velocity measurements. The systemic velocity of Cen A is $541 \pm 7 \mathrm{~km} \mathrm{~s}^{-1}$ (Hui et al. 1995), and its GCs have radial velocity measurements in the range 200-1000 km s ${ }^{-1}$ (see Peng. Ford, \& Freeman 2004a; Woodley et al. 2005, 2007). We are currently using roughly one-quarter to one-third of the entire estimated GC population of Cen A.

The X-ray point sources, found in the Chandra ACIS observations described in the previous section, were matched to our list of 340 GCs confirmed by radial velocity. We found 30 of these radially velocity confirmed GCs matched at least one X-ray source, a fraction of $8.7 \%$, within a search radius of $1.5^{\prime \prime}$, and 26 of these fell within a search radius of $1^{\prime \prime}$. Of the 75 GCs in the Woodley et al. (2007) catalog that are resolved by HST images, only 1 (GC0141) matched an X-ray source. This brings the matching fraction down to $7.5 \%$ for the total of 415 GCs. This range of search radius was chosen to be consistent with the accuracy of the positions of the GCs, determined from optical images, of $\pm 0.2^{\prime \prime}-$ 
0.3" (Harris, Harris \& Geisler 2004), as well as our X-ray point source positioning, which is better than $1^{\prime \prime}$. These $30 \mathrm{GC}$ X-ray sources, listed in Table 2, represent a three-fold improvement over the Minniti et al. (2004) study which found 11 matches to confirmed GCs.

Many additional GC candidates (not yet confirmed by velocity measurement or HST imaging) have been found in previous studies based on colors and morphologies alone (see Harris, Harris \& Geisler 2004; Gómez et al. 2006), as well as objects known to match X-ray sources in previous studies (Minniti et al. 2004; Peng, Ford, \& Freeman 2004a). Of these over $\sim 450$ GC candidates, we found 38 further matches with X-ray point sources within a search radius of $1.5^{\prime \prime}$, all of which also match within $1^{\prime \prime}$. These objects also are listed in Table 2. Because of the close physical association between LMXBs and GCs established in other galaxies, we regard these additional matches as very likely to be genuine GCs. In total, we find 67 probable GC X-ray sources in Cen A, doubling the number presented by Minniti et al. (2004).

Figure 1 displays the number of X-ray point sources that match the GCs within bins of $0.2^{\prime \prime}$ in search radius. We clearly see that most matches are within $0.2^{\prime \prime}-0.6^{\prime \prime}$. This trend is also seen in the offsets (in arcseconds) in RA and Dec. between the 67 matches that we found in this study, also shown in Fig. 1. The small offset between the two catalogs is well within our outer search radius of $1.5^{\prime \prime}$. However, we shifted the X-ray point sources by the mean offset of the matched RA and Dec. positions $\left(\mathrm{RA}=-0.2026^{\prime \prime}\right.$ and Dec. $\left.=0.0690^{\prime \prime}\right)$ and rematched the sources to all GCs and GC candidate objects. We obtained the same result.

We have performed tests on our matching program to determine the probability of false matches within our sample, assuming a search radius of $1.5^{\prime \prime}$. Shifting the positions of the $\mathrm{X}$-ray sources by $2^{\prime \prime}, 3^{\prime \prime}, 5^{\prime \prime}$, and $10^{\prime \prime}$ in right ascension and declination lead to 0,2 , 1 , and 1 false matches, respectively. We conclude that at most $1-2$ false matches probably exist in our list of 67 matches.

Although it is unusual to find more than one bright LMXB source within a single cluster (Sivakoff et al. 2007; Kundu et al. 2007), it is certainly known to occur (e.g. M15 in the Milky Way). One GC, GC0233, was found to match two X-ray point sources within 1.5", one of them being within a smaller radius of $0.5^{\prime \prime}$. It is possible that both X-ray sources are associated with this GC.

In Table 2, the first column lists the GC ID from Woodley et al. (2007), where clusters confirmed by either radial velocity or HST imaging have names beginning with "GC". The following columns are right ascension and declination (J2000), galactocentric radius in arcminutes, the various photometric indices $\left(B, V, I, T_{1},\left(C-T_{1}\right)\right.$, when known; magnitudes are not de-reddened), and metallicity $[\mathrm{Fe} / \mathrm{H}]$. The $B V I$ data are taken from Peng, Ford, \& Freeman 
(2004a) and the Washington photometry data is from Harris, Harris \& Geisler (2004). The metallicity $[\mathrm{Fe} / \mathrm{H}]$ was determined from the color $\left(C-T_{1}\right)_{0}$, using a conversion derived by Harris \& Harris (2002), which produces an uncertainty in $[\mathrm{Fe} / \mathrm{H}]$ of \pm 0.2 dex at the metalpoor end and \pm 0.07 dex at the metal-rich end for a typical photometric uncertainty of $\sigma\left(C-T_{1}\right) \simeq 0.1$, assuming a mean age of $\tau \sim 13$ Gyr.

The positions of the GCs and X-ray sources are shown in Figure 2, superposed on an optical image, and their radial positions in Figure 4. The GC X-ray sources are well within $10^{\prime}$ from the center of Cen A, limited by the extent of the Chandra fields.

Figure 3 is an adaptively smoothed, exposure corrected image from a combination of the observations tabulated in Table 1 , in the the $0.5-2.0 \mathrm{keV}$ band. The background has not been subtracted. The positions of the confirmed and candidate GCs are shown with the same color code as the previous figure.

While the GC system extends detectably farther out (Harris, Harris \& Geisler 2004), the majority are well within $15^{\prime}$ from the galaxy center. Thus if the X-ray point sources extend beyond the innermost regions of the galaxy, we would expect only a few more detections $(\lesssim 7)$ beyond $15^{\prime}$, assuming a matching percentage of $8.7 \%$ as we found above. Nevertheless, the outer regions of Cen A have not been searched as thoroughly as the inner regions for GCs, especially with spectroscopic confirmation. Similarly, the inner $5 \mathrm{kpc}$ of the galaxy has very few spectroscopically confirmed clusters due to the obscuration of the large dust lane extending across the center.

To estimate the number of GCs obscured in the vicinity of the dust lane, we show in Figure 5 the radial distribution of X-ray detections, all GCs, and GCs with associated X-ray sources in the dust lane and its vicinity. The dust lane is bounded roughly by an ellipse with semimajor axis of 4.2 and axial ratio 0.5 (Harris, Harris \& Geisler 2004). Within this region, there are 119 X-ray point sources and only 17 known GCs, 8 of which have radial velocity confirmation, many fewer clusters than the estimated $110 \pm 27$ (Harris, Harris \& Geisler 2004). The fraction of GCs in the $1^{\prime}$ annulus outside the dust lane region identified with LMXBs is 0.28. Applying this factor to the estimated number of clusters in the dust lane predicts $31 \pm 8$ matches, higher than the 12 matches we found. In other words, another $\sim 20$ GCs with LMXBs may remain to be optically identified in this inner region, though identifying them will be a considerable challenge. 


\section{Properties of the Globular Clusters matched with X-ray Sources}

\subsection{Optical colors}

Similar surveys of other early-type galaxies show that LMXBs are $\approx 3$ times more likely to be found in the redder, more metal-rich GCs. These include the studies of M87 (Jordán et al. 2004), NGC 4636 (Posson-Brown et al. 2007), NGC 1399 (Angelini et al. 2001), NGC 4472 (Kundu et al. 2002), and NGC 1553, NGC 4365, NGC 4649, NGC 4697 (Sarazin et al. 2003; Sivakoff et al. 2007) and samples of five (Kundu et al. 2007) and eleven (Sivakoff et al. 2007) early-type galaxies within $\sim 25 \mathrm{Mpc}$. In Cen A, Minniti et al. (2004) found that LMXBs also are found preferentially in redder GCs.

The distributions of the optical colors $((B-V),(V-I)$, and $(B-I))$ of the Cen A $\mathrm{GCs}$, and that of their derived metallicity $[\mathrm{Fe} / \mathrm{H}]$, are shown in Figure 6 for our Cen A GC sample. The entire GC population, shown as the open histogram, shows clear bimodality in all three colors. The GCs with associated X-rays, shown as the solid histogram, clearly show a preference for the redder GCs, as they do in other similar early-type galaxies. The hatched histogram includes all GCs within the same radial range as the outermost X-ray point source (corresponding to $16.5 \mathrm{kpc}$ ), and includes 314 out of 415 GCs.

The raw color-magnitude diagrams are shown in Figure 7, using the same notation, where filled circles denote GCs matched with X-ray point sources. Again, the bimodality of the GC system is apparent in all color indices. These color-magnitude plots, or the colorcolor plots (Figure 8) indicate that the GCs matched with X-ray sources, although redder, fall in general among the bulk of the GC population.

A few outlying X-ray sources in the $B V I$ diagram are quite faint, and could represent contaminant sources (foreground stars or background galaxies). Furthermore, a few objects, shown in the $(B-V)$ versus $V$ plot, show slightly bluer colors than the bulk of the confirmed population. All 12 matched X-ray sources with $(B-V)<0.5$ are unconfirmed GC candidates (see Table 2), and could therefore be younger star clusters, or simply contamination.

There are only two confirmed clusters, neither matched to an X-ray source, with ( $B-$ $V)<0.5$. These GCs (GC0084 and GC0103) have ages $\leq 1$ Gyr, as estimated by Peng, Ford, \& Freeman $(2004 \mathrm{~b})$. One of them (GC0103) appears to be located in a young blue tidal stream in the galaxy (Peng et al. 2002). It is possible that all of the GC candidates with associated X-ray binaries and very blue colors are young clusters, or perhaps even background galaxies with X-ray emission. Only spectroscopic follow-up can conclusively determine their true nature. The positions of these blue objects are clearly seen in Fig. 4 , 


\subsection{Dependence on Metallicity}

The $(B-I)$ color index, which is primarily sensitive to metallicity for old stellar populations (e.g. Worthey 1994), has been shown to clearly resolve the bimodal GC populations in several brightest cluster galaxies. In a study of eight galaxies, Harris et al. (2006) found that the blue and red cluster populations have $\langle B-I\rangle_{0}=1.64 \pm 0.03$ and $\langle B-I\rangle_{0}=2.06 \pm 0.05$ respectively, and internal dispersions of $\sigma_{B-I}=0.10 \pm 0.02$ and $\sigma_{B-I}=0.17 \pm 0.05$, respectively. Assuming a foreground absorption of $E(B-I)=0.273$ for Cen A, the transition between the metal-rich and metal-poor cluster populations is roughly at $(B-I) \simeq 2.072$. This division also agrees with the uncorrected color distributions of Peng, Ford, \& Freeman $(2004 \mathrm{~b})$. We have used $[\mathrm{Fe} / \mathrm{H}]$ data for confirmed clusters and $(B-I)$ colors when no $[\mathrm{Fe} / \mathrm{H}]$ data is available, to determine the fractions of metal-rich to metal-poor clusters hosting X-ray sources.

There is an obvious trend in the metallicity distribution of the GCs that contain X-ray point sources, when compared to the entire sample. The entire list of confirmed GCs contains 179 (53\%) metal-poor and 156 (47\%) metal-rich clusters of the 335 GCs with available metallicity data, closely matching previous studies (Harris, Harris \& Geisler 2004; Woodley et al. 2005). But of the confirmed GCs with X-ray sources, 6 (21\%) are metal-poor and $23(79 \%)$ are metal-rich clusters out of the 29 confirmed GC matches with available metallicity data. Of the additional candidate GC LMXBs, 16 (52\%) are metal-poor (blue) and 15 (48\%) are metal-rich (red) objects from the 32 candidate GCs with available metallicity data. From a straight combination of confirmed and candidate GCs, we therefore find that $22(37 \%)$ of LMXBs match metal-poor GCs and 38 (63\%) match metal-rich ones out of the 60 GCs with available data. We note though that some fraction of the GC candidates, particularly those with $(B-V)<0.5$ (see Figure 7), might be interlopers or very young clusters, so the fraction matched to the metal-rich clusters is probably a lower limit. We conclude therefore that the ratio of metal-rich to metal-poor GCs with associated LMXBs is $\gtrsim 1.7$. While previous observations of early-type galaxies gives a ratio of $\approx 3$, the scatter around this value is very

large (Sivakoff et al. 2007) and comfortably includes our lower limit. If we consider only the confirmed GCs, the ratio is 3 , more in line with the mean of the values found in previous studies.

\subsection{Dependence on GC Mass or GC Luminosity}

Finally, we explore the dependence of the incidence of point sources, which are predominantly LMXBs, on the luminosities (and indirectly on the masses) of the host GCs. Previous studies have found that X-ray point sources are preferentially found in optically more lu- 
minous GCs (e.g. Angelini et al. 2001; Kundu et al. 2003; Sarazin et al. 2003; Jordán et al. 2004; Sivakoff et al. 2007), while in the Galaxy and in M31 they have been noted to occur in the denser GCs (Bellazzini et al 1995). Because of the fact that the sizes of GCs do not correlate with their mass for masses $M \lesssim 2 \times 10^{6} M_{\odot}$ (McLaughlin 2000; Jordán et al. 2005) a preference of LMXBs in massive clusters translates into a preference for denser GCs, which in the picture of dynamically formed LMXB progenitors is the most fundamental property. In the left panel of Figure 9, we plot the distribution of the $I$-band magnitudes of all the GCs (open histogram) along with the magnitude distribution of those that match point sources (filled histogram). As before, the hatched histogram corresponds to all GCs out to $16.5 \mathrm{kpc}$. While LMXBs are hosted by GCs having luminosities in a wide range, it is clear that the distribution of GCs that host an LMXB does not follow the parent GC distribution. Instead, the distribution is much flatter as a consequence of a higher fraction of massive GCs hosting LMXBs.

In the right-hand panel of Fig. 9, in each one-magnitude bin, we have calculated the total I-band luminosity of all GCs by assuming the adopted distance of 3.8 Mpc (for GCs with no $I$ magnitude, we use an estimate from their $T 1$ magnitude). We plot the number of matched X-ray point sources per unit GC luminosity (for all GCs with $r<16.5 \mathrm{kpc}$ ) in each bin. The errors reflect Poisson errors in the number of GCs. We have made an approximate correction for completeness, by comparing the number of GCs in each magnitude bin to those predicted by the GC luminosity function and radial distribution of GCs in Harris et al. (2006). This correction is very large and uncertain fainter than $I=19.87$, which is indicated by the dashed vertical line.

The number of matched point sources (or, equivalently, the number of matched GCs) per unit GC luminosity is consistent with being constant across the luminosity range of our GC sample. We determine that GCs in Cen $\mathrm{A}$ host on average $\approx 0.4$ LMXBs per $10^{6} L_{\odot, I}$ (uncorrected luminosities). In other words, and consistent with previous studies (e.g. Sarazin et al. 2003; Jordán et al. 2004; Sivakoff et al. 2007), we find that the number of X-ray point sources per unit GC luminosity is roughly constant or, equivalently, that the probability of a GC hosting an LMXB is roughly proportional to its mass. Sarazin et al. (2003) found in their sample of four galaxies that GCs host $\approx 0.15$ LMXBs per $10^{6} L_{\odot, I}$. Their lower value, compared to the one found here, can be understood as the result of the fact that their LMXB samples were restricted to higher X-ray luminosities than ours (their $\mathrm{X}$-ray point source sample has mostly $L_{X} \gtrsim 10^{38} \mathrm{erg} \mathrm{s}^{-1}$ ). Therefore, they probe a smaller fraction of the LMXB population which naturally implies a lower observed number of LMXBs per unit GC mass. 


\section{Properties of the matched X-ray point Sources}

The four Chandra/ACIS observations explored in this work together cover almost all (about 310 sq.arcmin) of the circle of radius 10 arcmin centered on the galaxy (see Fig. 2, also Voss \& Gilfanov (2006)). According to the Moretti et al. (2003) compilation of pointsource counts various ROSAT and Chandra Deep Field surveys, the number of background sources (mostly AGN) expected down to a $0.5-2 \mathrm{keV}$ flux limit of $f_{X}>1 \times 10^{-15}$ erg s $\mathrm{s}^{-1}$ $\mathrm{cm}^{-2}$ and $f_{X}>5 \times 10^{-16} \mathrm{erg} \mathrm{s}^{-1} \mathrm{~cm}^{-2}$ within this area of the sky is 67 and 104 respectively. We find 153 and $185 \mathrm{X}$-ray point sources brighter than these flux limits respectively within this circle. Here we use the $0.5-2 \mathrm{keV}$ flux for each point source, taking the mean value for sources that are detected in multiple observations.

If we take a smaller circle, that of radius 5 arcmin, from the centre, the background counts down to the flux limit of $f_{X}>1 \times 10^{-15} \mathrm{erg} \mathrm{s}^{-1} \mathrm{~cm}^{-2}$ and $f_{X}>5 \times 10^{-16} \mathrm{erg} \mathrm{s}^{-1} \mathrm{~cm}^{-2}$ within this radius are 17 and 26, whereas we detect 92 and 114 point sources down to these flux limits respectively.

In Figure 10a, we plot the luminosity function (LF) of the matched X-ray point sources (solid histogram) within $5^{\prime}$ of the center of Cen A. There are 36 matched point sources within this region. Of these, 30 have mean $0.5-2 \mathrm{keV}$ fluxes more than $f_{X}>5 \times 10^{-16} \mathrm{erg} \mathrm{s}^{-1} \mathrm{~cm}^{-2}$. Down to this flux limit, there are 84 sources that are not matched with any confirmed or candidate GC. The unmatched sources are represented by the red unbroken histogram in the same plot.

It can be argued that a significant fraction of the unmatched X-ray sources may lie in the dust lane region, where there are far fewer detected GCs than X-ray point sources (see Fig. 2) . To investigate this, we define the dust lane region to be an ellipse with a semi-major axis of $4.5^{\prime}$, parallel to the dust lane, and a semi-minor axis of $1.2^{\prime}$, thus occupying $22 \%$ (but in the core of the galaxy) of the surveyed area within the $5^{\prime}$ circle (see Fig. 2). Outside this dust lane region, shown as the purple dashed curve, 44 of the 84 unmatched sources with flux

$f_{X}>5 \times 10^{-16} \mathrm{erg} \mathrm{s}^{-1} \mathrm{~cm}^{-2}$ exist. This shows that if one excludes the dust lane region, the numbers of matched and unmatched point sources are comparable, down to a luminosity of about $L_{X}=8.6 \times 10^{36} \mathrm{erg} \mathrm{s}^{-1}$. For the distributions of these 30 and 44 objects respectively, a Kolmogorov-Smirnov (K-S) test shows that the probability that the two subsamples could be drawn from the same population is about $54 \%$.

The black dash-dotted and dotted curves represent the expected counts of background Xray sources (mostly AGN and background galaxies) estimated from the Moretti et al. (2003) compilation, normalized to the area covered by the solid red histogram and dashed purple histograms respctively. Brighter than $\sim 5 \times 10^{-16} \mathrm{erg} \mathrm{s}^{-1} \mathrm{~cm}^{-2}$, there are 26 background 
sources expected within the $5^{\prime}$ circle, but only 16 if one considers the area within the circle excluding the dust lane. So outside the dust lane, within $5^{\prime}, 36 \%(=16 / 44)$ of the unmatched sources are expected to be background ones. It must also be noted that Voss \& Gilfanov (2006) find that in the Cen A field, the density of X-ray point sources is significantly higher than the average values found by studies by that of Moretti et al. (2003). Even with the enhanced background counts, the background point source population does not account for at least half of the unmatched sources above a flux of $\sim 5 \times 10^{-16} \mathrm{erg} \mathrm{s}^{-1} \mathrm{~cm}^{-2}$, even outside the dust lane region, where the GC catalogue is expected to be reasonably complete.

In Figure 10b, we plot the same curves for the X-ray point sources within $10^{\prime}$ of the center of Cen A. There are 53 matched point sources within this region with flux more than $f_{X}>5 \times 10^{-16} \mathrm{erg} \mathrm{s}^{-1} \mathrm{~cm}^{-2}$, whereas there are 133 sources that are not matched with any confirmed or candidate GC, 93 of which are outside the dust lane region. Unlike the above case of $<5$ arcmin objects, the subsamples of the matched 53 sources and unmatched 93 sources have a $1.7 \%$ chance of being drawn from the same population, according to the K-S test. This shows that outside the $5^{\prime}$ circle, the detected point sources are increasingly not associated with the galaxy. Indeed, of these 93 unmatched sources out to 10 arcmin, 62 are expected to be background sources from the Moretti et al. (2003) counts, and higher according to the Voss \& Gilfanov (2006) study.

\section{Concluding remarks}

We have presented a study of the connection between GCs and LMXBs using a groundbased catalog of GCs and an X-ray point source list constructed using four Chandra/ACIS Xray observations of the nearby early-type galaxy Cen A, obtained over a four-year period, each with an exposure time of 37-50 ks. Of the $353 \mathrm{X}$-ray point sources detected on one or more of these Chandra fields, 67 are found to be hosted by known GCs or GC candidates. Thirty of these matches correspond to GCs confirmed by radial velocity, and 1 match corresponds to a resolved GC by HST imaging. Even in the central heavily obscured dust lane, we find matches between the two populations, although the optical samples are significantly incomplete in that region.

As in many other early-type galaxies (e.g. Peng et al. 2006), the GCs in Cen A have a large range in metallicity and a bimodal color distribution. A three-to-one majority of the matched X-ray point sources are seen to lie in the redder, more metal-rich GCs: specifically, $156 / 335$ (44\%) of the confirmed GCs are in the metal-rich subpopulation, while 23/29 (79\%) of the X-ray point sources matched to confirmed GCs lie in these clusters. We also note a possible double X-ray point source detection in the radially velocity confirmed GC0233. 
Also in agreement with previous studies, we find that luminous GCs are most likely to host LMXBs. Quantitatively, the number of LMXBs per unit GC mass is roughly constant. The lack of correlation between GC size and mass implies that denser GCs are more likely to host LMXBs, as expected if most GC LMXB are formed dynamically by tidal capture or exchange processes in GC cores.

Our expanded sample of GC-LMXB identifications in Cen A gives further support for a consistent picture which has been emerging from combined optical and Chandra observations of early-type galaxies. Important factors determining the presence of LMXBs in GCs which can be easily determined from ground-based observations are their mass, which is a reflection of a more fundamental dependence on dynamical formation rates (Jordán et al. 2007), and their metallicity. The latter is a bona fide effect which seems not to follow from any possible trends of GC structural properties or age with metallicity.

The X-ray luminosity function of the LMXBs that are found in GCs and of those that are not matched with GCs are found to have the same slope, revealing similar underlying populations. In fact, if one samples the areas of the galaxy that have more complete coverage for both the LMXB and GC populations (away from the dust lanes and within 10' of the galaxy center), half of the LMXBs are found within GCs.

However, there is some indication that, towards the faint end of the luminosity function, the fraction of LMXBs found in GCs is far lower than at the more luminous end, but it is not clear whether this is due to incompleteness effects at the faint end. Cen A offers a unique opportunity to study this question because of its proximity, and we will probe the LMXB/GC connection with our upcoming deep observations to X-ray luminosities unattainable in more

distant early-type galaxies. In addition, we plan to use high-resolution optical imaging material, from both ground based telescopes and from the HST/ACS, to probe the structural properties of the GCs with and without LMXBs, as well as the Chandra spectral properties of the X-ray sources.

This work was supported by NASA contracts NAS8-39073 and NAS8-03060, the Chandra Science Center and the Smithsonian Institution. KAW and WEH acknowledge financial support from the Natural Sciences and Engineering Research Council of Canada.

\section{REFERENCES}

Angelini, L., Loewenstein, M., \& Mushotzky, R.F. 2001, ApJ, 557, L35 
Beasley, M. A., Sharples, R. M., Bridges, T. J., Hanes, D. A., Zepf, S. E., Ashman, K. M., Geisler, D. 2000, MNRAS, 318, 1249

Beasley, M. et al. 2007 MNRAS, submitted

Bellazzini, M., Pasquali, A. Federici, L., Ferraro, F.R., \& Fusi Pecci, F. 1995, ApJ, 439, 687

Cohen, J. G., Blakeslee, J. P., \& Ryzhov, A. 1998, ApJ, 496, 808

Di Stefano, R., Kong, A.K.H., VanDalfsen, M.L., Harris, W.E., Murray, S.S., \& Delain, K.M. 2003, ApJ, 599, 1067

Dufour, R.J., van den Bergh, S., Harvel, C.A., Martins, D.H., Schiffer, F.H., Talbot, R.J., Talent, D.L., \& Wells, D.C. 1979, AJ, 84, 284

Fabbiano, G. 2006, ARA\&A, 44, 323

Fabbiano, G., Brassington, N. J., Zezas, A. et al. 2007, astro-ph/0710.5126

Fabian, A. C., Pringle, J. E., \& Rees, M. J. 1975, MNRAS, 172, 15P

Fan, Z., Ma, J., Zhou, X,. Chen, J., Jiang, Z., \& Wu, Z. 2005, PASP, 117, 1236

Feigelson, E. D., Schreier, E. J., Delvaille, J. P., Giacconi, R., Grindlay, J. E., \& Lightman, A. P. 1981, ApJ, 251, 31

Ferrarese, L., Mould, J. R., Stetson, P. B., Tonry, J. L., Blakeslee, J. P., Ajhar, E. A., 2007, ApJ, 654, 186

Gómez, M., Geisler, D., Harris, W.E., Richtler, T., Harris, G.L.H., \& Woodley, K.A. 2006, A\&A, 447, 877

Gómez, M. \& Woodley, K. A. 2007, ApJ, 670, L105

Graham, J. A., \& Phillips, M. M. 1980, ApJ, 239, L97

Grindlay, J. E. 1987, The Origin and Evolution of Neutron Stars, 125, 173

Grindlay, J. E. 1993, The Globular Cluster-Galaxy Connection, 48, 156

Hardcastle, M. J., Kraft, R. P., Sivakoff, G. R. et al. 2007, astro-ph/0710.1277

Harris, G.L.H., Geisler, D., Harris, H.C., \& Hesser, J.E. 1992, AJ, 104, 613

Harris, W.E., Harris, G.L.H., Barmby, P., McLaughlin, D.E., \& Forbes, D.A. 2006, AJ, 132, 2187 
Harris, W.E., Whitmore, B.C., Karakla, D., Okoń, W., Baum, W.A., Hanes, D.A., \& Kavelaars, J.J. 2006, ApJ, 636, 90H

Harris, G.L.H., Harris, W.E., \& Geisler, D. 2004, AJ, 128, 723

Harris, W.E. \& Harris, G.L.H. 2002, AJ, 123, 3108

Harris, W.E., Harris, G.L.H., Holland, S.T., \& McLaughlin, D.E. 2002, AJ, 124, 1435

Haynes, R. F., Cannon, R. D., \& Ekers, R. D. 1983, Proceedings of the Astronomical Society of Australia, 5, 241

Hills, J. G. 1976, MNRAS, 175, 1P

Hui, X., Ford, H.C., Freeman, K.C., \& Dopita, M.A. 1995, ApJ, 449, 592

Irwin, J. A. 2005, ApJ, 631, 511

Israel, F. P. 1998, A\&A Rev., 8, 237

Ivanova, N. 2006, ApJ, 653, L137

Jordán, A. 2004, ApJ, 613, L117

Jordán, A., Sivakoff, G. R., McLaughlin, D. E., et al. 2007, ApJ, 671, L117

Jordán, A., Côté, P., West, M. J., \& Marzke, R. O. 2002, ApJ, 576, L113

Jordán, A., Côté, P., Ferrarese, L., Blakeslee, J.P., Mei, S., Merritt, D., Milosavljević, M., Peng, E.W., Tonry, J.L., \& West, M.J. 2004, ApJ, 613, 279

Jordán, A., Côté, P., Blakeslee, J.P., Ferrarese, L., McLaughlin, D.E., Mei, S., Peng, E.W., Tonry, J.L., Merritt, D., Milosavljević, M., Sarazin, C.L., Sivakoff, G.R., \& West, M.J. 2005, ApJ, 634, 1002

Juett, A. M. 2005, ApJ, 621, L25

Karachentsev, I.D. et al. 2007, AJ, 133, 504

Katz, J. I. 1975, Nature, 253, 698

Kim, E., Kim, D.-W., Fabbiano, G., Lee, M. G., Park, H. S., Geisler, D., \& Dirsch, B. 2006, ApJ, 647, 276

Kraft, R. P., et al. 2000, ApJ, 531, L9 
Kraft, R.P., Kregenow J.M., Forman, W.R., Jones, C., \& Murray, S.S. 2001, ApJ, 560, 675

Kraft, R. P., Forman, W. R., Jones, C., Murray, S. S., Hardcastle, M. J., \& Worrall, D. M. 2002, ApJ, 569, 54

Kraft, R. P., Hardcastle, M. J., Forman, W. R., Jones, C., Murray, S. S., \& Worrall, D. M. 2003, New Astronomy Review, 47, 625

Kraft, R. P., Vázquez, S. E., Forman, W. R., Jones, C., Murray, S. S., Hardcastle, M. J., Worrall, D. M., \& Churazov, E. 2003, ApJ, 592, 129

Kundu, A., \& Whitmore, B.C. 2001, AJ, 121, 2950

Kundu, A., Maccarone, T.J., \& Zepf, S.E. 2002, ApJ, 574, L5

Kundu, A., Maccarone, T.J., Zepf, S.E., \& Puzia, T.H. 2003, ApJ, 589, L1

Kundu, A., Maccarone, T. J., \& Zepf, S.E. 2007, ApJ, 662, 525

Maccarone, T. J., Kundu, A., \& Zepf, S. E. 2004, ApJ, 606, 430

McLaughlin, D.E. 2000, ApJ, 539, 618

McLaughlin, D.E., Barmby, P., Harris, W.E., Harris, G.L.H., \& Forbes, D.A. 2007, MNRAS, submitted

Minniti, D., Rejkuba, M., Funes, J. G., \& Akiyama, S. 2004, ApJ, 600, 716

Moretti, A., Campana, S., Lazzati, D., \& Tagliaferri, G. 2003, ApJ, 588, 696

Peng, E.W., Ford, H.C., \& Freeman, K.C. 2004a, ApJS, 150, 367

Peng, E.W., Ford, H.C., \& Freeman, K.C. 2004b, ApJ, 602, 705

Peng, E.W., Ford, H.C., Freeman, K.C., \& White, R.L. 2002, AJ, 124, 3144

Peng, E.W., Jordán, A., Côte, P., Blakeslee, J.P., Ferrarese, L., Mei, S., West, M.J., Merritt, D., Milosavljevic, M., \& Tonry, J.L. 2006, ApJ, 639, 95

Pooley, D. et al. 2003, ApJ, 591, L131

Posson-Brown, J., Raychaudhury, S., Forman, W., Hank Donnelly, R., \& Jones, C. 2007, submitted to ApJ(astro-ph/0605308)

Puzia, T H., Kissler-Pattig, M., Brodie, J. P., \& Huchra, J. P. 1999, AJ, 118, 2734 
Raychaudhury, S. 1989, Nature, 342, 251

Rejkuba, M. 2001, A\&A, 369, 812

Rejkuba, M., Greggio, L., Harris, W.E., Harris, G.L.H., \& Peng, E.W. 2005, ApJ, 631,262

Rosenberg, A., Saviane, I., Piotto, G., \& Aparicio, A. 1999, AJ, 118, 2306

Sarazin, C. L., Irwin, J. A., \& Bregman, J. N. 2001, ApJ, 556, 533

Sarazin, C.L., Kundu, A., Irwin, J.A., Sivakoff, G.R., Blanton, E.L., \& Randall, W.E. 2003, ApJ, 595, 743

Schiminovich, D., van Gorkom, J. H., van der Hulst, J. M., \& Kasow, S. 1994, ApJ, 423, L101

Sivakoff, G.R., Jordán, A., Sarazin, C.L., Blakeslee, J.P., Côté, P., Ferrarese, L., Juett, A.M., Mei, S., \& Peng, E.W. 2007, ApJ, 660, 1246

Turner, T. J., George, I. M., Mushotzky, R. F., \& Nandra, K. 1997, ApJ, 475, 118

Voss, R., \& Gilfanov, M. 2006, A\&A, 447, 71

Voss, R., \& Gilfanov, M. 2007, A\&A, 468, 49

White, N. E., \& Angelini, L. 2001, ApJ, 561, L101

White, R. E., III, Sarazin, C. L., \& Kulkarni, S. R. 2002, ApJ, 571, L23

Woodley, K.A., Harris, W.E., \& Harris, G.L.H. 2005, AJ, 129, 2654

Woodley, K.A. 2006, AJ, 132, 2424

Woodley, K. A., Harris, W. E., Beasley, M. A., Peng, E. W., Bridges, T. J., Forbes, D. A., \& Harris, G. L. H. 2007, AJ, 134, 494

Worthey, G. 2004, ApJS, 95, 107 
Table 1. Summary of Chandra/ACIS observations of Centaurus A used here

\begin{tabular}{cccc}
\hline \hline OBSID & Date & Instrument & Cleaned Exposure Time \\
\hline 00316 & 5 Dec 99 & ACIS-I & $35724 \mathrm{~s}$ \\
00962 & 17 May 00 & ACIS-I & $36505 \mathrm{~s}$ \\
02978 & 3 Sep 02 & ACIS-S & $44592 \mathrm{~s}$ \\
03965 & 14 Sep 03 & ACIS-S & $49517 \mathrm{~s}$ \\
\hline
\end{tabular}


Table 2. Globular Clusters in Cen A (NGC 5128) with X-ray Sources

\begin{tabular}{|c|c|c|c|c|c|c|c|c|c|}
\hline Cluster ${ }^{\mathrm{a}}$ & $\begin{array}{c}\text { RA } \\
(\mathrm{J} 2000)\end{array}$ & $\begin{array}{c}\text { Dec } \\
(\mathrm{J} 2000)\end{array}$ & $\begin{array}{l}\text { Radius } \\
\text { (arcmin) }\end{array}$ & $\begin{array}{c}B \\
(\mathrm{mag})\end{array}$ & $\begin{array}{c}V \\
(\mathrm{mag})\end{array}$ & $\begin{array}{c}I \\
(\mathrm{mag})\end{array}$ & $\begin{array}{c}T_{1} \\
(\mathrm{mag})\end{array}$ & $\left(C-T_{1}\right)$ & {$[\mathrm{Fe} / \mathrm{H}]$} \\
\hline $\mathrm{GC} 0074^{\mathrm{b}}$ & 132454.35 & -425324.8 & 9.84 & 18.24 & 17.25 & 16.02 & 16.68 & 1.788 & -0.6732 \\
\hline GC0109 & 132503.13 & -425625.1 & 6.51 & 19.94 & 18.93 & 17.67 & 18.36 & 1.880 & -0.5088 \\
\hline GC0120 & 132505.02 & -425715.0 & 5.68 & - & - & - & 16.83 & 1.863 & -0.5379 \\
\hline $\mathrm{GC} 0123^{\mathrm{b}}$ & 132505.72 & -431030.7 & 10.18 & - & - & - & 17.36 & 1.984 & -0.3429 \\
\hline GC0129b & 132507.62 & -430115.2 & 3.66 & - & - & - & 17.45 & 2.026 & -0.2818 \\
\hline $\mathrm{GC} 0134^{\mathrm{b}}$ & 132509.19 & -425859.2 & 4.00 & 18.65 & 17.71 & 16.55 & - & - & - \\
\hline $\mathrm{GC} 0137^{\mathrm{b}}$ & 132510.25 & -425509.5 & 6.78 & 20.47 & 19.46 & 18.21 & 18.86 & 1.927 & -0.4248 \\
\hline GC0138 ${ }^{b}$ & 132510.27 & -425333.1 & 8.23 & 18.79 & 17.80 & 16.55 & 17.25 & 1.895 & -0.4769 \\
\hline GC0141 & 132511.05 & -430132.3 & 3.05 & - & - & - & - & - & - \\
\hline GC0158b & 132514.24 & -430723.5 & 6.71 & 20.63 & 19.55 & 18.21 & 18.95 & 2.066 & -0.2269 \\
\hline $\mathrm{GC}^{2} 191^{\mathrm{b}}$ & 132522.19 & -430245.6 & 1.90 & 18.17 & 17.21 & 16.03 & 16.51 & 1.858 & -0.5466 \\
\hline GC0205b & 132527.97 & -430402.2 & 2.89 & 20.23 & 19.18 & 17.90 & 18.60 & 2.036 & -0.2678 \\
\hline $\mathrm{GC} 0210^{\mathrm{b}}$ & 132529.43 & -425809.9 & 3.00 & 20.58 & 19.49 & 18.12 & 18.94 & 2.067 & -0.2257 \\
\hline $\mathrm{GC} 0217^{\mathrm{b}}$ & 132530.29 & -425934.8 & 1.64 & - & - & - & - & - & - \\
\hline $\mathrm{GC} 0225^{\mathrm{b}}$ & 132531.60 & -430002.8 & 1.32 & 20.99 & 19.93 & 18.43 & - & - & - \\
\hline GC0230 & 132532.42 & -425850.2 & 2.47 & 19.86 & 18.86 & 17.64 & 18.33 & 1.801 & -0.6489 \\
\hline $\mathrm{GC} 0231^{\mathrm{b}}$ & 132532.80 & -425624.4 & 4.83 & 19.43 & 18.64 & 17.64 & 18.22 & 1.287 & -1.8618 \\
\hline $\mathrm{GC} 0233^{\mathrm{b},}$ & ${ }^{c} 132532.88$ & -430429.2 & 3.48 & 20.44 & 19.37 & 18.12 & 18.74 & 2.104 & -0.1727 \\
\hline $\mathrm{GC} 0246^{\mathrm{b}}$ & 132535.16 & -425301.0 & 8.25 & 21.31 & 20.25 & 18.94 & 19.75 & 1.973 & -0.3534 \\
\hline GC0249 & 132535.50 & -425935.2 & 2.12 & 20.18 & 19.17 & 17.94 & - & - & - \\
\hline GC0260 & 132538.61 & -425919.6 & 2.71 & 19.83 & 18.93 & 17.79 & 18.44 & 1.616 & -1.0249 \\
\hline
\end{tabular}


Table 2-Continued

\begin{tabular}{|c|c|c|c|c|c|c|c|c|c|}
\hline Cluster $^{\mathrm{a}}$ & $\begin{array}{c}\text { RA } \\
(\mathrm{J} 2000)\end{array}$ & $\begin{array}{c}\text { Dec } \\
(\mathrm{J} 2000)\end{array}$ & $\begin{array}{l}\text { Radius } \\
\text { (arcmin) }\end{array}$ & $\begin{array}{c}B \\
(\mathrm{mag})\end{array}$ & $\begin{array}{c}V \\
(\mathrm{mag})\end{array}$ & $\begin{array}{c}I \\
(\mathrm{mag})\end{array}$ & $\begin{array}{c}T_{1} \\
(\mathrm{mag})\end{array}$ & $\left(C-T_{1}\right)$ & {$[\mathrm{Fe} / \mathrm{H}]$} \\
\hline GC0266 $6^{\mathrm{b}}$ & 132539.88 & -430501.9 & 4.49 & 18.42 & 17.53 & 16.43 & 16.89 & 1.603 & -1.0539 \\
\hline $\mathrm{GC} 0275^{\mathrm{b}}$ & 132542.00 & $-43 \quad 1042.2$ & 9.91 & 20.08 & 19.26 & 18.22 & 18.94 & 1.414 & -1.5015 \\
\hline $\mathrm{GC} 281^{\mathrm{b}}$ & 132543.23 & -425837.4 & 3.81 & 20.46 & 19.42 & 18.18 & 18.92 & 1.879 & -0.5105 \\
\hline GC0295 & 132546.59 & -425703.0 & 5.37 & 20.89 & 19.87 & 18.63 & 19.41 & 1.853 & -0.5553 \\
\hline $\mathrm{GC} 0314^{\mathrm{b}}$ & 132550.40 & -425802.3 & 5.20 & 20.23 & 19.42 & 18.45 & 19.06 & 1.290 & -1.8476 \\
\hline $\mathrm{GC} 0320^{\mathrm{b}}$ & 132552.74 & -430546.4 & 6.52 & 18.76 & 17.87 & 16.77 & 17.40 & 1.576 & -1.1150 \\
\hline GC0330 & 132554.58 & -425925.4 & 5.22 & 18.29 & 17.22 & 15.95 & 16.69 & 1.904 & -0.4686 \\
\hline $\mathrm{GC} 0347^{\mathrm{b}}$ & 132600.81 & -430940.1 & 10.46 & 21.09 & 20.09 & 18.91 & 19.57 & 1.846 & -0.5676 \\
\hline $\mathrm{GC} 0378^{\mathrm{b}}$ & 132610.58 & -425342.7 & 10.81 & 19.38 & 18.43 & 17.26 & 17.96 & 1.691 & -0.8644 \\
\hline $\mathrm{GC} 0384^{\mathrm{b}}$ & 132619.66 & -430318.6 & 9.76 & 19.68 & 18.74 & 17.57 & 18.28 & 1.633 & -0.9876 \\
\hline BTC136 & 132501.16 & -431159.0 & 11.87 & - & - & - & 19.68 & 2.155 & -0.1161 \\
\hline BTC317 & 132545.63 & -430115.5 & 3.30 & 19.84 & 18.97 & 18.02 & - & - & - \\
\hline Minniti03 & 132538.18 & -425815.3 & 3.48 & - & - & - & - & - & - \\
\hline Minniti05 & 132533.95 & -425859.5 & 2.45 & 18.21 & 17.73 & - & 18.62 & 1.751 & -0.7440 \\
\hline Minniti17 & 132525.76 & -430056.0 & 0.40 & 21.17 & 20.83 & 19.37 & - & - & - \\
\hline Minniti23 & 132520.86 & -430053.7 & 1.26 & 22.70 & 21.89 & 20.90 & - & - & - \\
\hline Minniti26 & 132542.13 & -430319.7 & 3.44 & 21.05 & 20.14 & 19.11 & - & - & - \\
\hline Minniti33 & 132537.46 & -430131.4 & 1.84 & 19.12 & - & - & - & - & - \\
\hline Minniti40 & 132532.02 & -430231.6 & 1.60 & 20.32 & 19.09 & 17.76 & - & - & - \\
\hline Minniti44 & 132529.45 & -430108.4 & 0.34 & 19.65 & 18.70 & 17.39 & - & - & - \\
\hline Minniti53 ${ }^{\mathrm{d}}$ & 132527.49 & -430128.4 & 0.33 & 17.78 & 17.57 & - & - & - & - \\
\hline
\end{tabular}


Table 2-Continued

\begin{tabular}{|c|c|c|c|c|c|c|c|c|c|}
\hline Cluster $^{\mathrm{a}}$ & $\begin{array}{c}\text { RA } \\
(\mathrm{J} 2000)\end{array}$ & $\begin{array}{c}\text { Dec } \\
(\mathrm{J} 2000)\end{array}$ & $\begin{array}{l}\text { Radius } \\
\text { (arcmin) }\end{array}$ & $\begin{array}{c}B \\
(\mathrm{mag})\end{array}$ & $\begin{array}{c}V \\
(\mathrm{mag})\end{array}$ & $\begin{array}{c}I \\
(\mathrm{mag})\end{array}$ & $\begin{array}{c}T_{1} \\
(\mathrm{mag})\end{array}$ & $\left(C-T_{1}\right)$ & {$[\mathrm{Fe} / \mathrm{H}]$} \\
\hline Minniti55 & 132525.59 & -430209.7 & 1.08 & 22.70 & 21.71 & 20.48 & - & - & - \\
\hline Minniti57 & 132525.15 & -430126.8 & 0.54 & 20.82 & 19.44 & 18.05 & - & - & - \\
\hline Minniti71 & 132520.09 & -430310.1 & 2.45 & 21.31 & 20.19 & 18.88 & 19.62 & 1.925 & -0.4344 \\
\hline Minniti74 & 132518.50 & $-4301 \quad 16.3$ & 1.67 & 19.47 & 18.20 & - & - & - & - \\
\hline Minniti80 & 132514.03 & -430242.9 & 2.94 & - & - & - & 19.85 & 1.359 & -1.6584 \\
\hline Minniti82 & 132512.89 & -430114.7 & 2.69 & 21.17 & 20.00 & 18.66 & - & - & - \\
\hline Minniti208 & 132522.36 & -425717.1 & 3.98 & - & - & - & 16.10 & 3.251 & -0.0206 \\
\hline Minniti216 & 132507.65 & -425630.2 & 5.91 & - & - & - & - & - & - \\
\hline K-007 & 132458.17 & -430949.2 & 10.21 & 20.99 & 20.54 & 19.95 & 20.34 & 0.629 & -4.1622 \\
\hline K-041 & 132511.98 & -425713.3 & 4.86 & 19.96 & 19.09 & 17.98 & 18.57 & 1.564 & -1.1427 \\
\hline K-047 & 132513.82 & -425331.1 & 8.04 & 21.73 & 20.64 & 18.94 & 19.95 & 1.967 & -0.3686 \\
\hline K-073 & 132522.67 & -425501.6 & 6.19 & 21.70 & 21.11 & 20.39 & 20.64 & 0.423 & -5.0574 \\
\hline $\mathrm{K}-110$ & 132529.10 & -430746.2 & 6.63 & 22.55 & 21.23 & 19.73 & 20.44 & 2.664 & 0.2205 \\
\hline K-116 & 132531.05 & -431107.1 & 9.99 & 20.02 & 19.86 & 19.19 & 19.56 & 0.498 & -4.7219 \\
\hline K-141 & 132534.05 & -431031.1 & 9.45 & 21.29 & 20.10 & 18.77 & 19.67 & 0.000 & - \\
\hline $\mathrm{K}-159$ & 132539.08 & -425653.6 & 4.74 & 20.35 & 19.90 & 19.26 & 19.62 & 0.803 & -3.4708 \\
\hline $\mathrm{K}-182$ & 132546.34 & -430310.2 & 3.98 & 22.48 & 21.34 & 19.65 & - & 0.000 & - \\
\hline K-192 & 132548.71 & -430323.4 & 4.46 & 19.96 & 18.72 & 17.41 & 18.14 & 2.419 & 0.1217 \\
\hline K-201 & 132553.75 & -431155.6 & 11.79 & 21.85 & 20.62 & 19.04 & 19.98 & 2.216 & -0.0490 \\
\hline K-204 & 132555.13 & $-4301 \quad 18.3$ & 5.03 & 21.86 & 21.37 & 20.73 & 21.22 & 1.024 & -2.6780 \\
\hline K-207 & 132556.87 & -430044.4 & 5.36 & 21.04 & 20.58 & 19.63 & 20.31 & 0.442 & -4.9713 \\
\hline
\end{tabular}


Table 2-Continued

\begin{tabular}{cccccccccc}
\hline \hline Cluster $^{\mathrm{a}}$ & $\begin{array}{c}\mathrm{RA} \\
(\mathrm{J} 2000)\end{array}$ & $\begin{array}{c}\text { Dec } \\
(\mathrm{J} 2000)\end{array}$ & $\begin{array}{c}\text { Radius } \\
(\mathrm{arcmin})\end{array}$ & $\begin{array}{c}B \\
(\mathrm{mag})\end{array}$ & $\begin{array}{c}V \\
(\mathrm{mag})\end{array}$ & $\begin{array}{c}I \\
(\mathrm{mag})\end{array}$ & $\begin{array}{c}T_{1} \\
(\mathrm{mag})\end{array}$ & $\left(C-T_{1}\right)$ & {$[\mathrm{Fe} / \mathrm{H}]$} \\
\hline $\mathrm{K}-209$ & 132557.42 & -425341.6 & 9.23 & 19.45 & 19.14 & 18.34 & 18.54 & 0.570 & -4.4101 \\
$\mathrm{~K}-216$ & 132558.71 & -430430.7 & 6.61 & 19.26 & 19.02 & 18.49 & 19.19 & -0.297 & -8.8381 \\
$\mathrm{~K}-224$ & 132611.86 & -430243.2 & 8.24 & 21.56 & 21.18 & 20.02 & 20.87 & 0.408 & -5.1258 \\
$\mathrm{~K}-235$ & 132620.42 & -425946.4 & 9.75 & 21.60 & 21.20 & 20.45 & 21.08 & 0.345 & -5.4180 \\
\hline
\end{tabular}

${ }^{a}$ Cluster Names: BTC objects are candidate GCs from Harris, Harris \& Geisler (2004), Minniti objects are candidate clusters from Minniti et al. (2004), and the remaining candidate GCs, denoted by the prefix K (with no radial velocity measurement) are from Peng, Ford, \& Freeman (2004a).

${ }^{\mathrm{b}}$ GCs with radial velocity measurement listed as an average weight of all previous measurements in Woodley et al. (2007).

${ }^{\mathrm{c}}$ GC0233 matched both X-ray point source 55 and 56 within $1.5^{\prime \prime}$, and one of them within $0.5^{\prime \prime}$.

d Minniti et al. (2004) suggest that Minniti53 is spectroscopically confirmed from Harris et al. (1992), yet in this study, we do not find that the position of Minniti53 matches any previously known GC with a radial velocity measurement within a search radius of $2^{\prime \prime}$. We list this object as a candidate GC. 

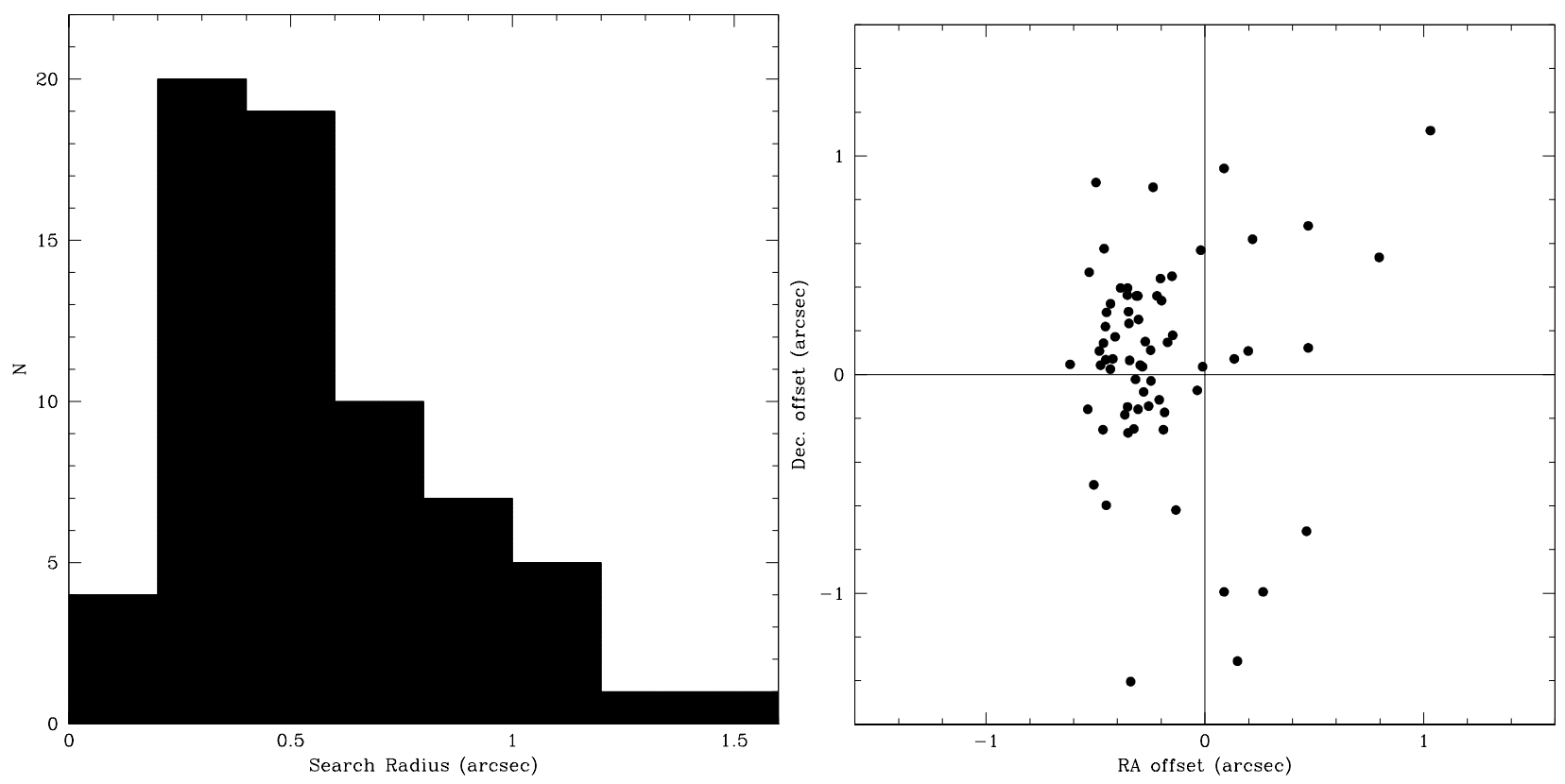

Fig. 1.- (a) The number of X-ray point sources matching GCs binned within search radii of $0.2^{\prime \prime}$.(b) The RA and Dec. offsets of the 67 matched X-ray point sources to GCs. A mean offset between the two catalogs of RA $=-0.2026^{\prime \prime}$ and Dec. $=0.0690^{\prime \prime}$ exists. We have used these offsets to align the X-ray point source catalog with the GC positions in order to search for LMXBs. 


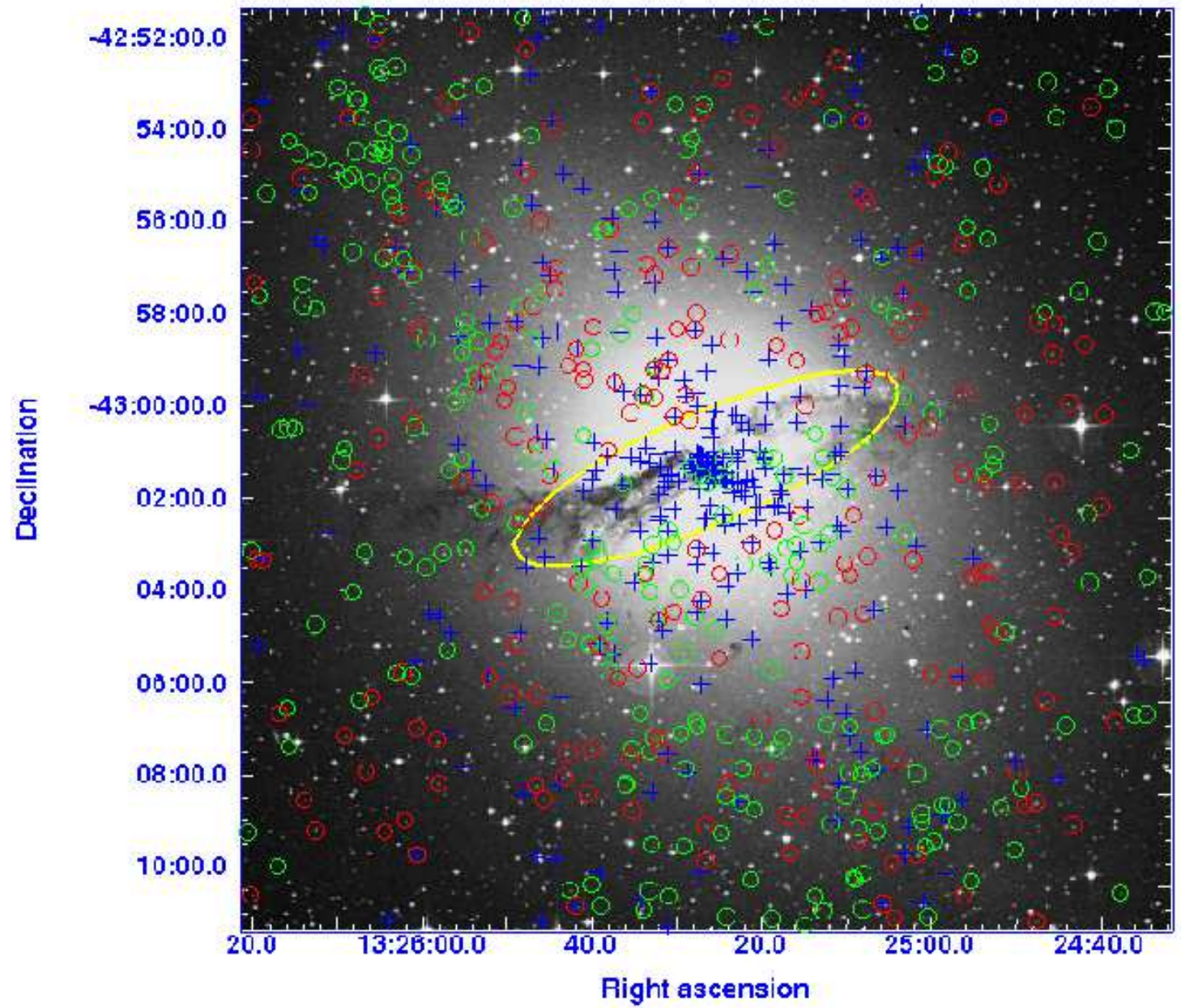

Fig. 2.- A optical image of the early-type galaxy Centaurus A (NGC 5128), together with the X-ray point sources (blue crosses), the confirmed GC population (red open circles) and the GC candidates unconfirmed by spectroscopy (green open circles) in the inner region of Cen A. The optical image is from the IIIa-J blue UKSTU plates, obtained from the STSCI Digitized Sky Survey (http://archive.stsci.edu/). 


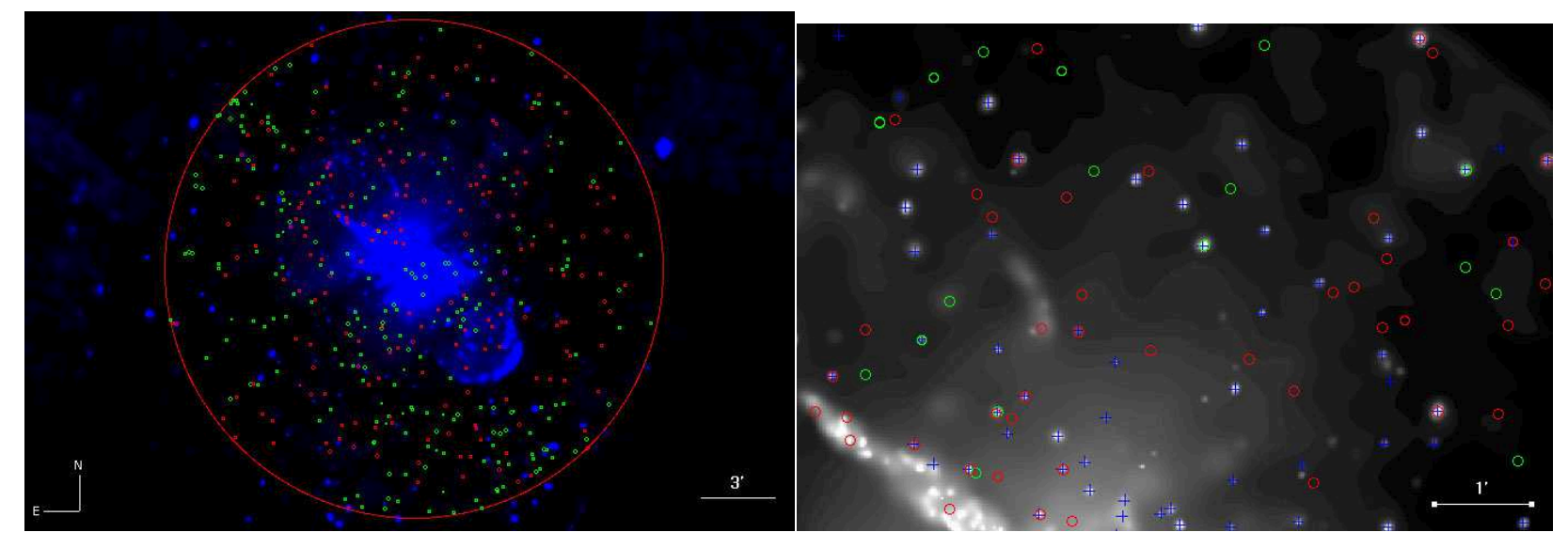

Fig. 3.- (a) A soft X-ray image (0.5-2.0 keV) of NGC 5128, adaptively smoothed and exposure corrected combined image with the confirmed GC population (red open circles) and the GC candidates unconfirmed by spectroscopy (green open circles), within 10 arcmin of the center of the galaxy (marked as a circle). (b) A close-up of a part of the galaxy, where the detected X-ray point sources above the flux limit of this paper are shown as blue crosses as well, with a GCs shown as circles as before. 


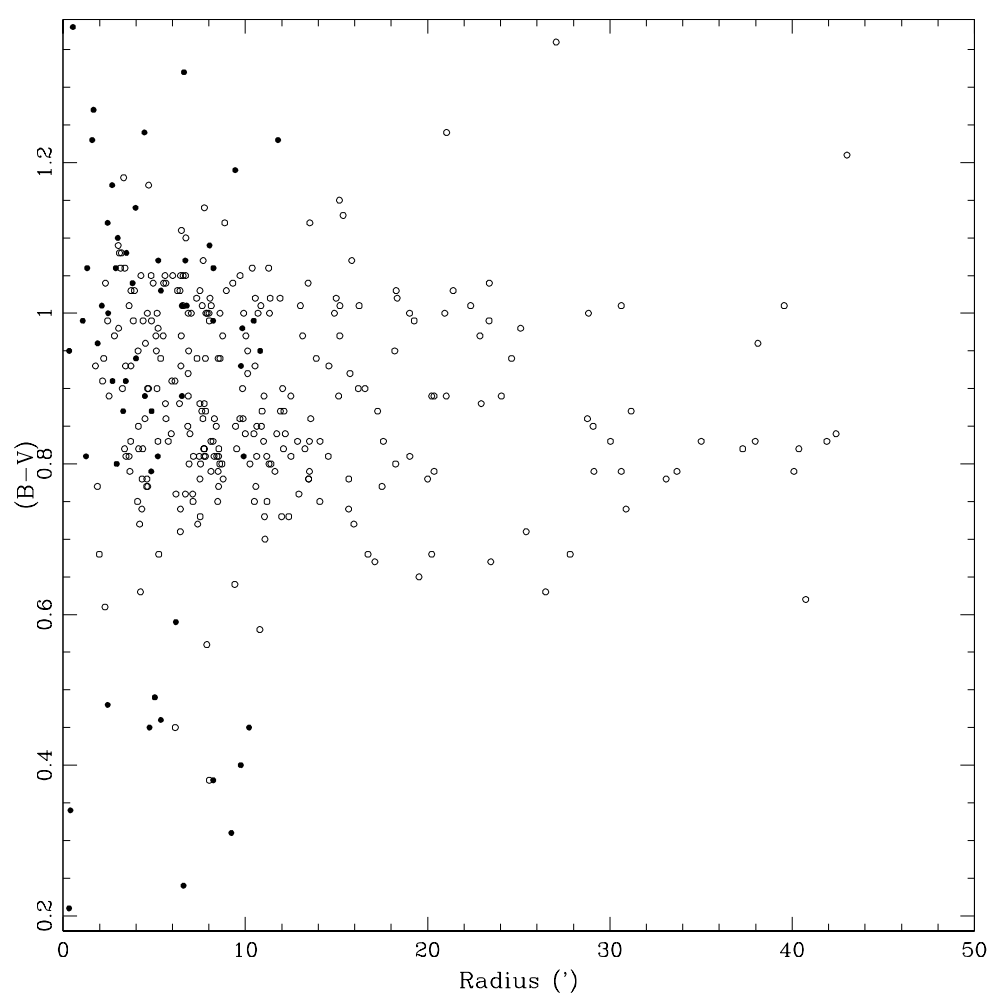

Fig. 4.- The radial distribution of the confirmed sample of GCs in Cen A (open circles) and the GCs and candidates with X-ray matches (closed circles) in $(B-V)$. GC candidates with X-ray matches with a color of $(B-V)<0.5$ are too blue to be old GCs. 


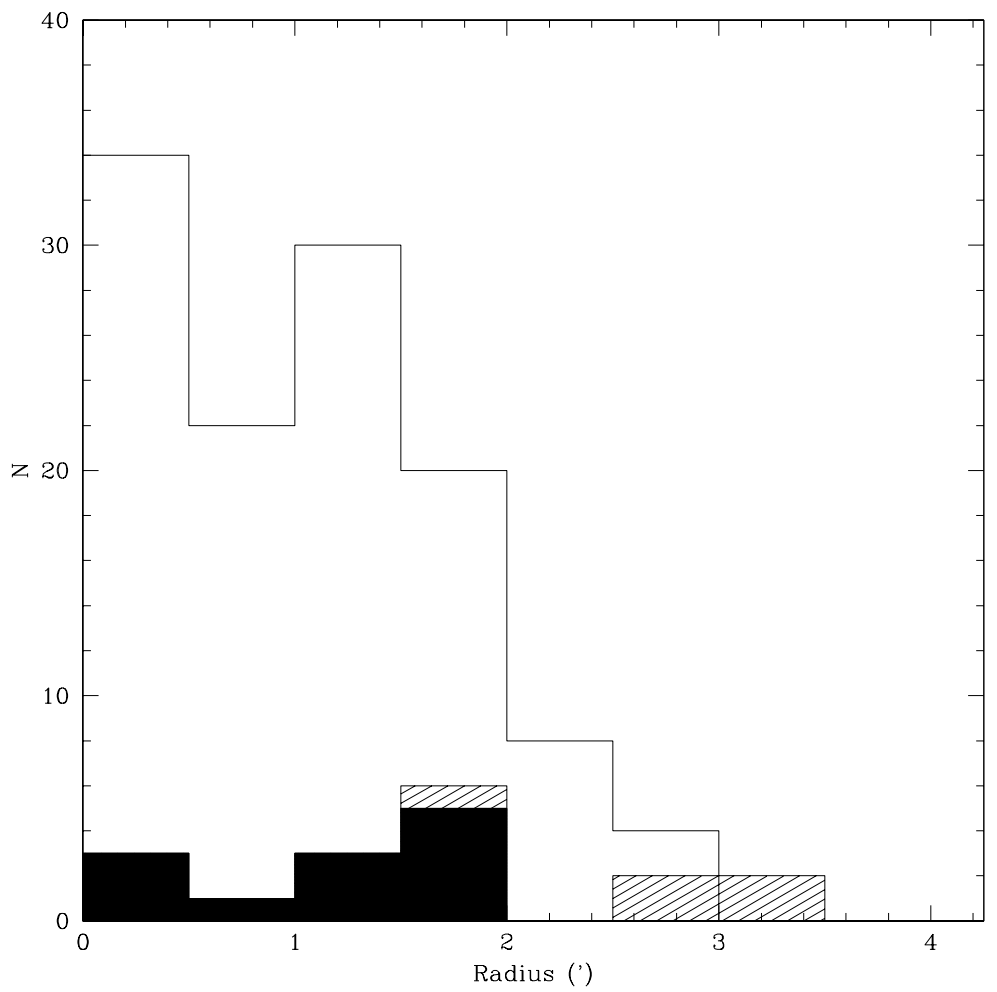

Fig. 5.- The radial distribution of X-ray point sources and GCs in the inner 4'.2 occupied by the Cen A dust lane. The open histogram is for all X-ray point sources, the hatched histogram is known GCs, and the solid histogram is all GC X-ray sources enclosed in the exclusion ellipse (see text). 

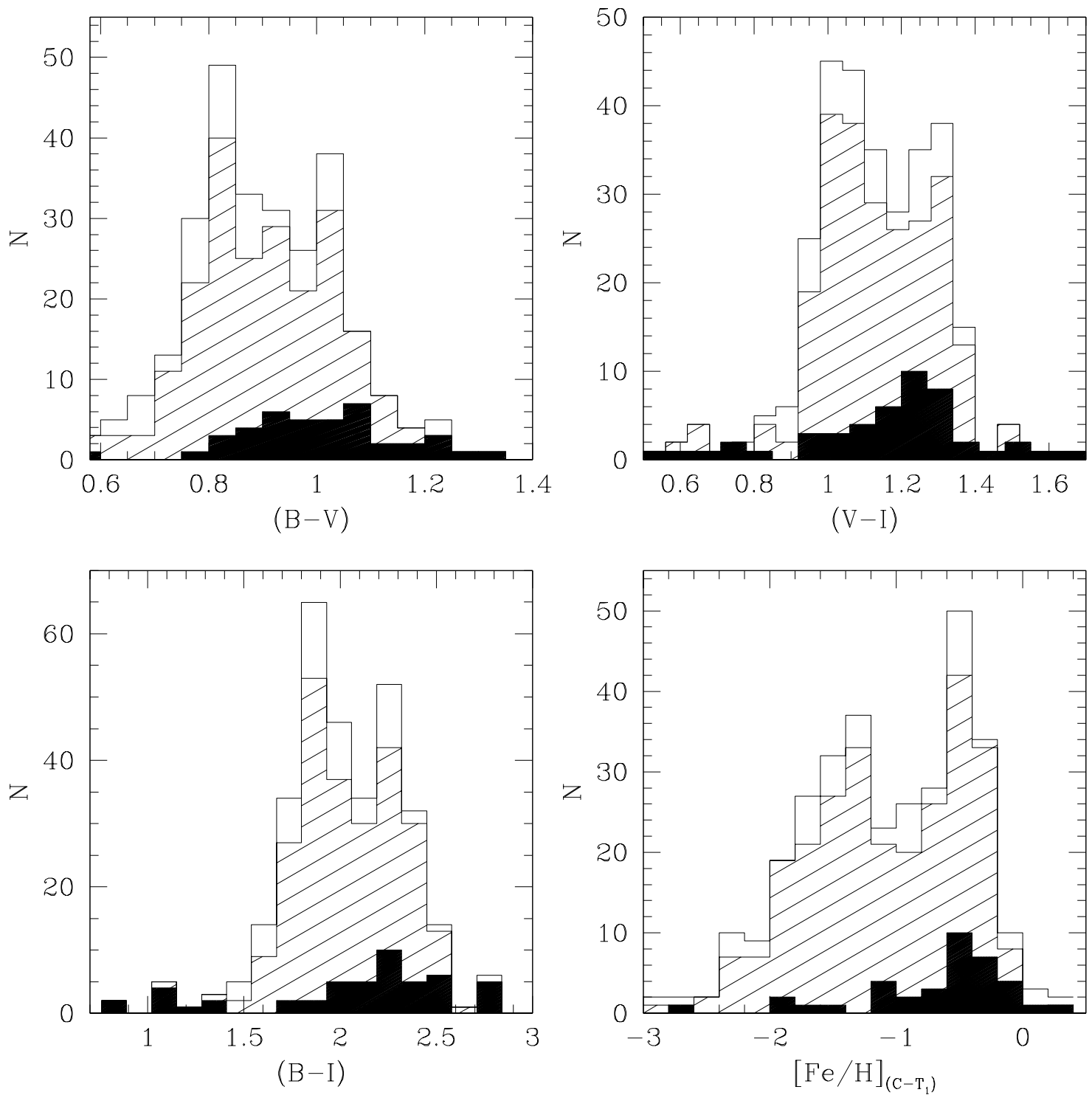

Fig. 6. - The color distributions and the metallicity distribution function of the entire sample of GCs in Cen A (open histograms), and the GCs matched with an X-ray source (solid histograms) in (upper left) $(B-V)$, (upper right) $(V-I)$, (lower left) $(B-I)$, and (lower right) $[\mathrm{Fe} / \mathrm{H}]_{\left(\mathrm{C}-\mathrm{T}_{1}\right)}$. The hatched histograms represent all GCs within $16.5 \mathrm{kpc}$ of the center of the galaxy, the radius out to which LMXBs are detected in this study. 

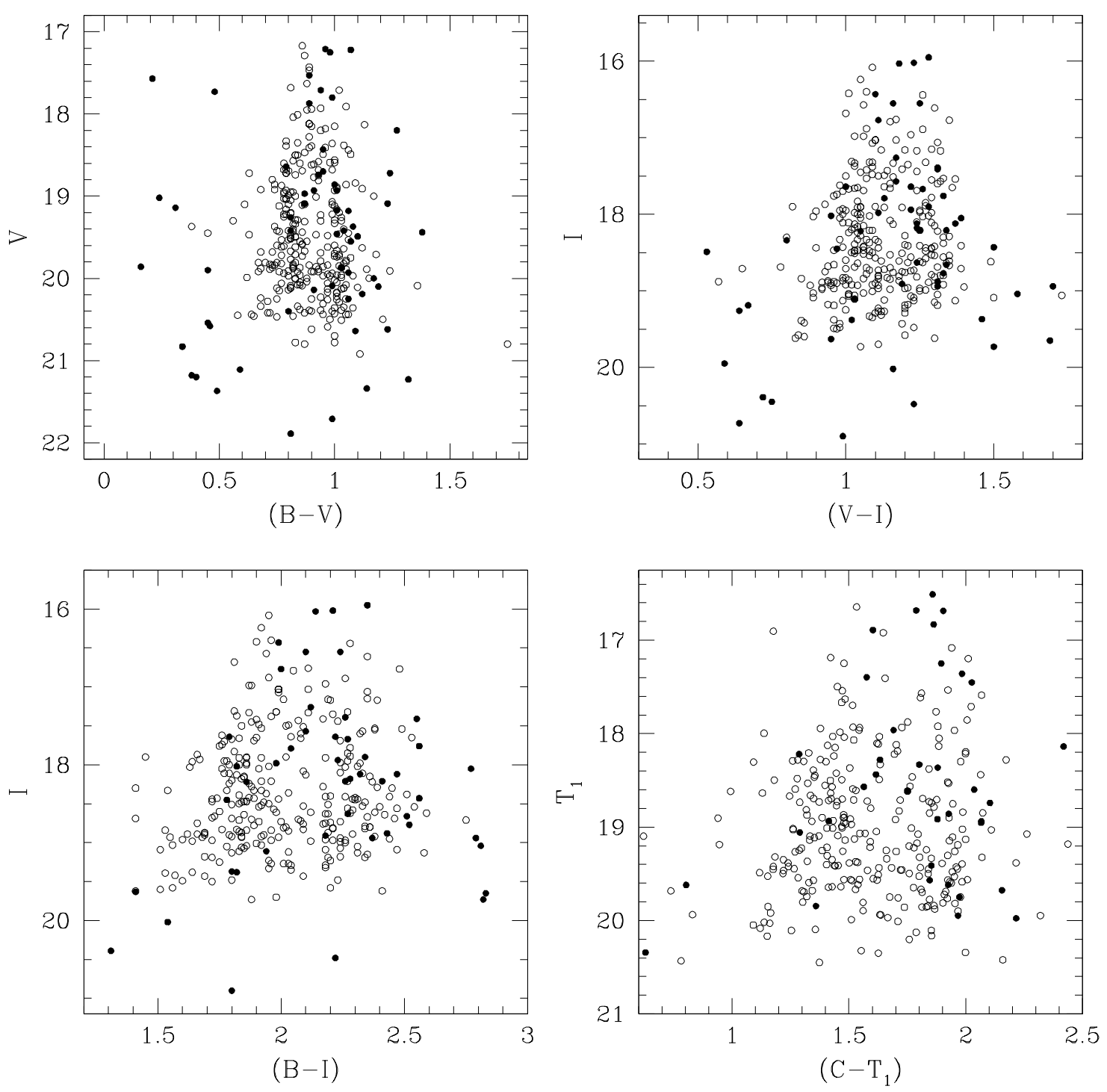

Fig. 7. - The color-magnitude diagrams of the sample of GCs in Cen A (open circles) and the GCs and candidates with X-ray matches (closed circles) in (upper left) $(B-V)$ versus $V$ magnitude, (upper right) $(V-I)$ versus $I$ magnitude, (lower left) $(B-I)$ versus $I$ magnitude, and (lower right) $\left(C-T_{1}\right)$ versus $T_{1}$ magnitude. 

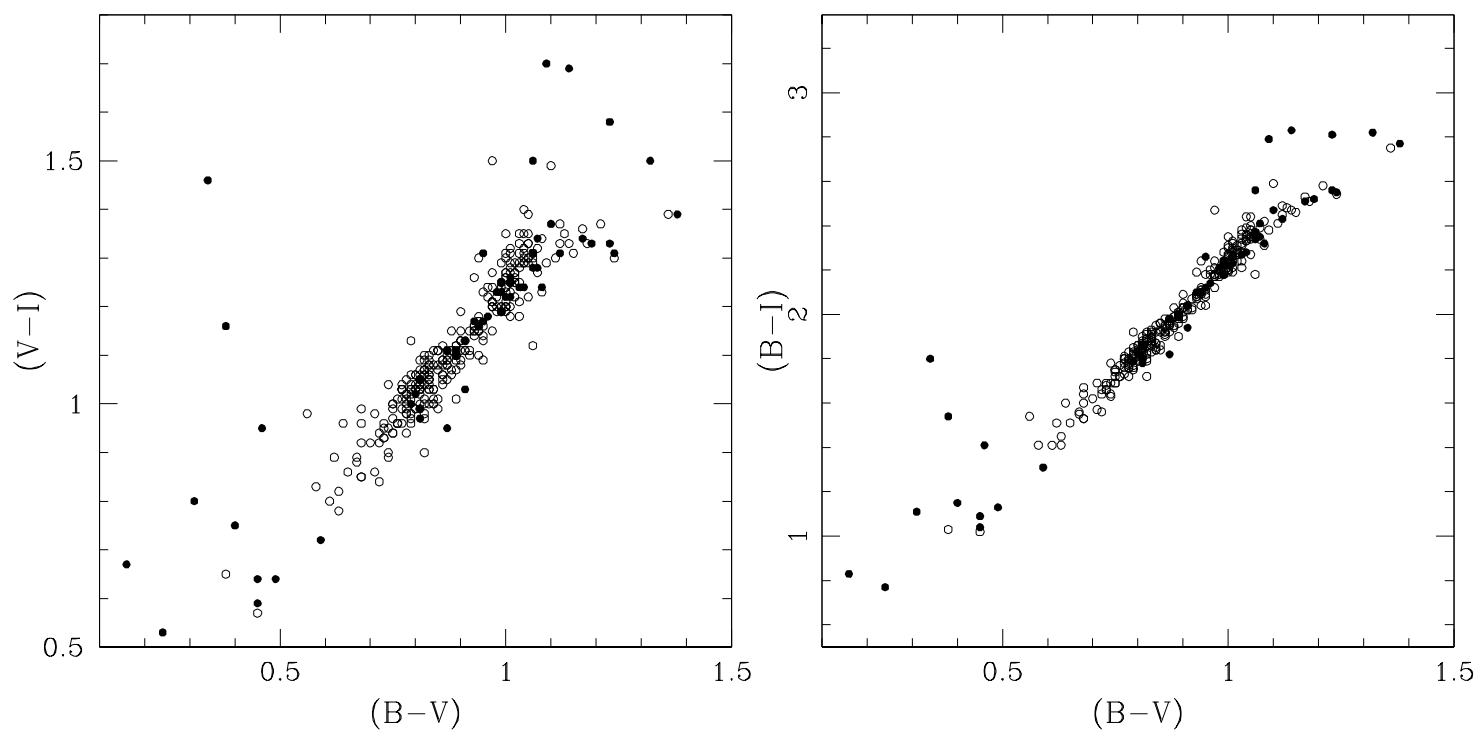

Fig. 8. - The color-color diagrams of the sample of GCs in Cen A (open circles) and the GCs and candidates with X-ray matches, in (left) $(B-V)$ versus $(V-I)$ and $(r i g h t)(B-V)$ versus $(B-I)$. 

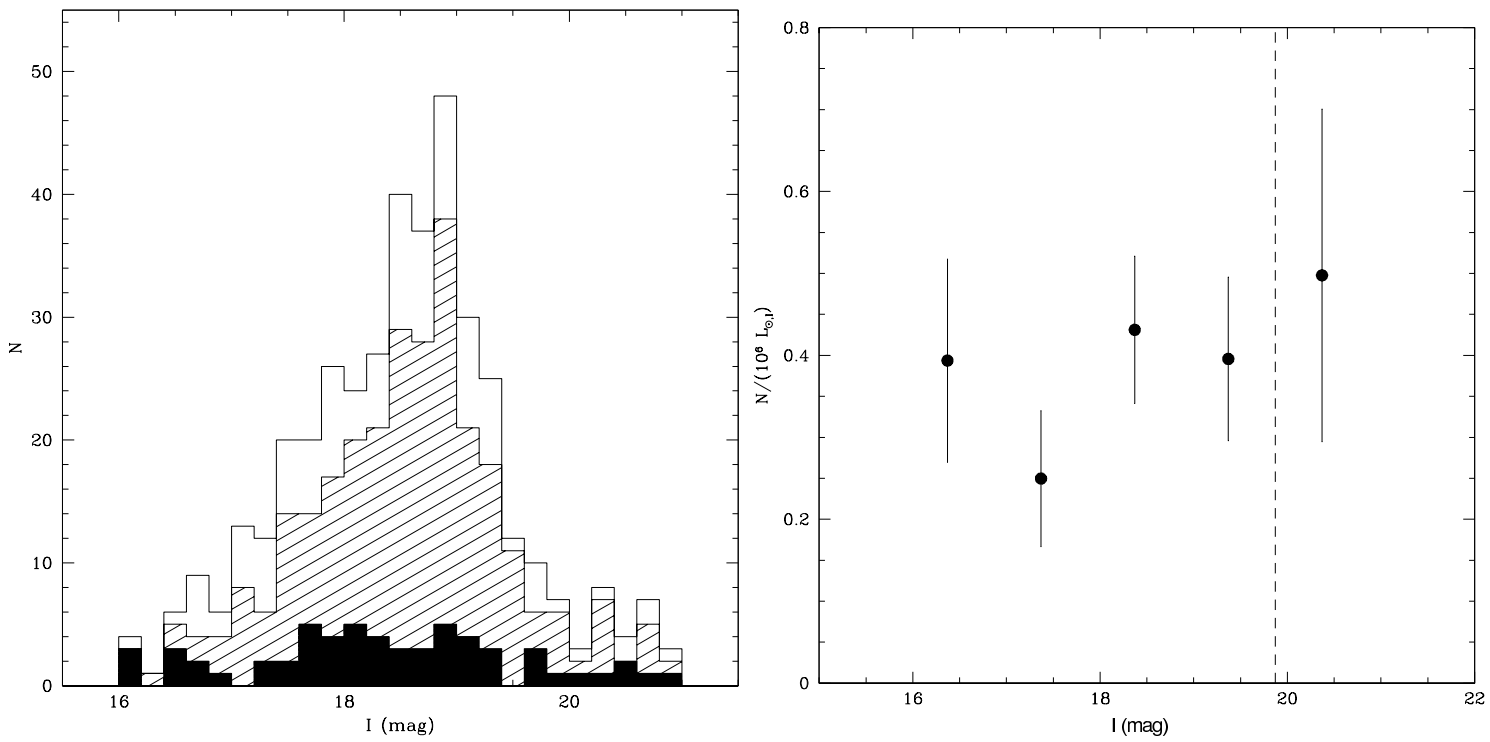

Fig. 9.- (a) The distribution of total apparent I magnitude of the GCs used in this study, where the open histogram represents all confirmed GCs in Cen A, and the solid histogram shows GCs matched with an X-ray source. The hatched histogram represents all GCs within $16.5 \mathrm{kpc}$ of the center of the galaxy, the radius out to which LMXBs are detected in this study. There is no obvious trend of finding LMXBs with GC luminosity. (b) The number of matched X-ray point sources per unit GC luminosity (for all GCs with $r<16.5 \mathrm{kpc}$ ) as a function of $I$ magnitude of the GCs. In each one-magnitude bin, the total $I$-band luminosity of all GCs is calculated by assuming them to be all at the adopted distance of the galaxy, and an estimated correction is made for incompleteness (see \$4). The number of matched LMXBs per unit light is independent of GC luminosity. The sample is seriously incomplete fainter than $I=19.87$, indicated by the dashed vertical line. 

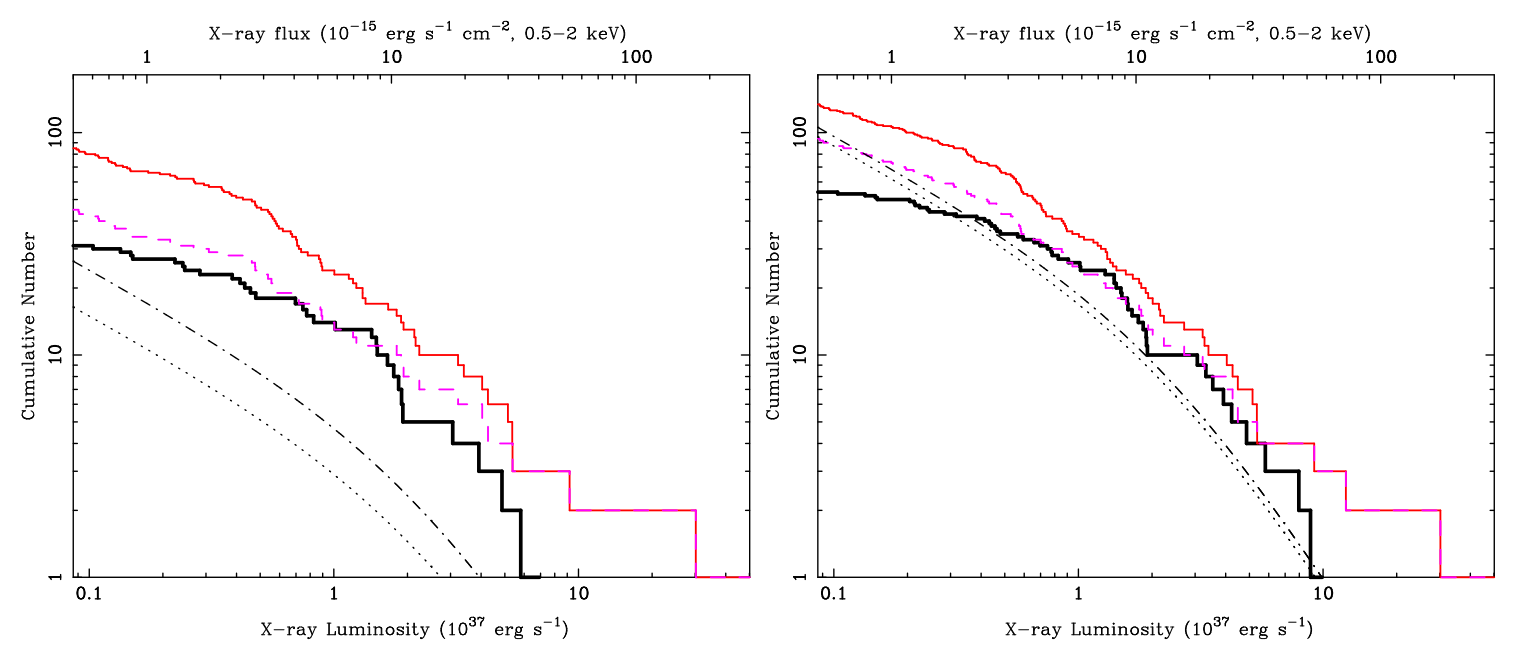

Fig. 10.- (Left:) The luminosity function (LF) of all 30 X-ray point sources, brighter than $5 \times 10^{-16} \mathrm{erg} \mathrm{s}^{-1}(0.5-2 \mathrm{keV})$, found within $5^{\prime}$ of the center of Cen A (solid histogram), that are found to match a GC. For comparison, we also plot the LF of all the X-ray point sources that did not match GCs (red solid histogram, $N=84$ ). Of these, the unmatched source within $5^{\prime}$, but outside the dust lane region $\left(N=44\right.$ brighter than $\left.5 \times 10^{-16} \mathrm{erg} \mathrm{s}^{-1}\right)$ are shown as the dashed purple histogram. The black dash-dotted and dotted curves represent the expected counts of backgound X-ray sources (mostly AGN and background galaxies) estimated from deep blank sky surveys, compiled by (Moretti et al. 2003), normalized to the area covered by the solid red histogram and dashed purple histogram respctively. Clearly the background sources do not account for almost half of the unmatched sources above a flux of $\sim 5 \times 10^{-16} \mathrm{erg} \mathrm{s}^{-1} \mathrm{~cm}^{-2}$, even outside the dust lane region where the GC catalogue is expected to be reasonably complete. In this plot, we use the $0.5-2 \mathrm{keV}$ luminosity of each point source, taking the average value for sources that are detected in multiple observations. (Right:) The same curves, but for all sources found within $10^{\prime}$ of the center of Cen A. Outside the 5 arcmin region, the fraction of unmatched sources accounted for by background sources is subtantially larger. 

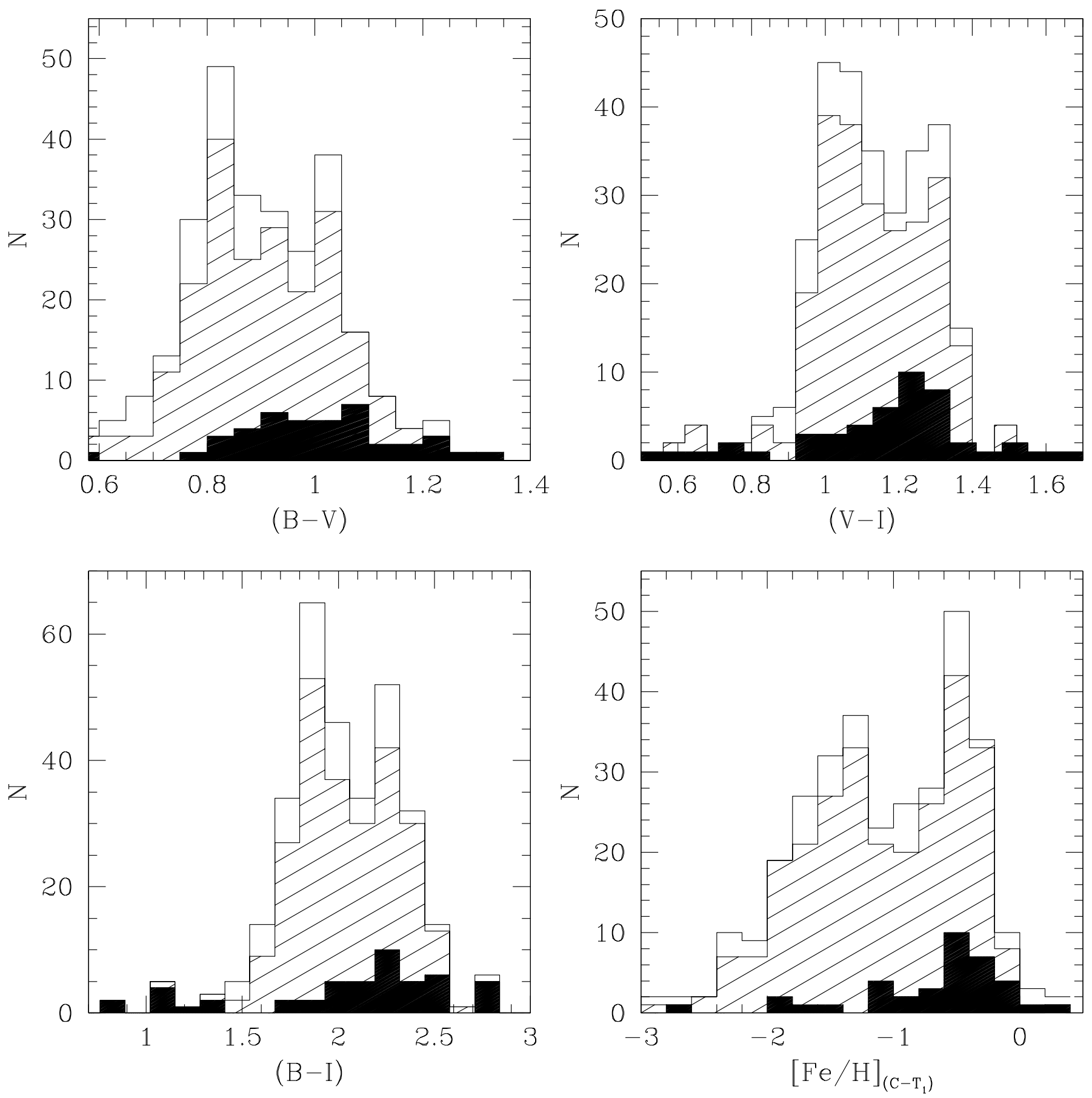


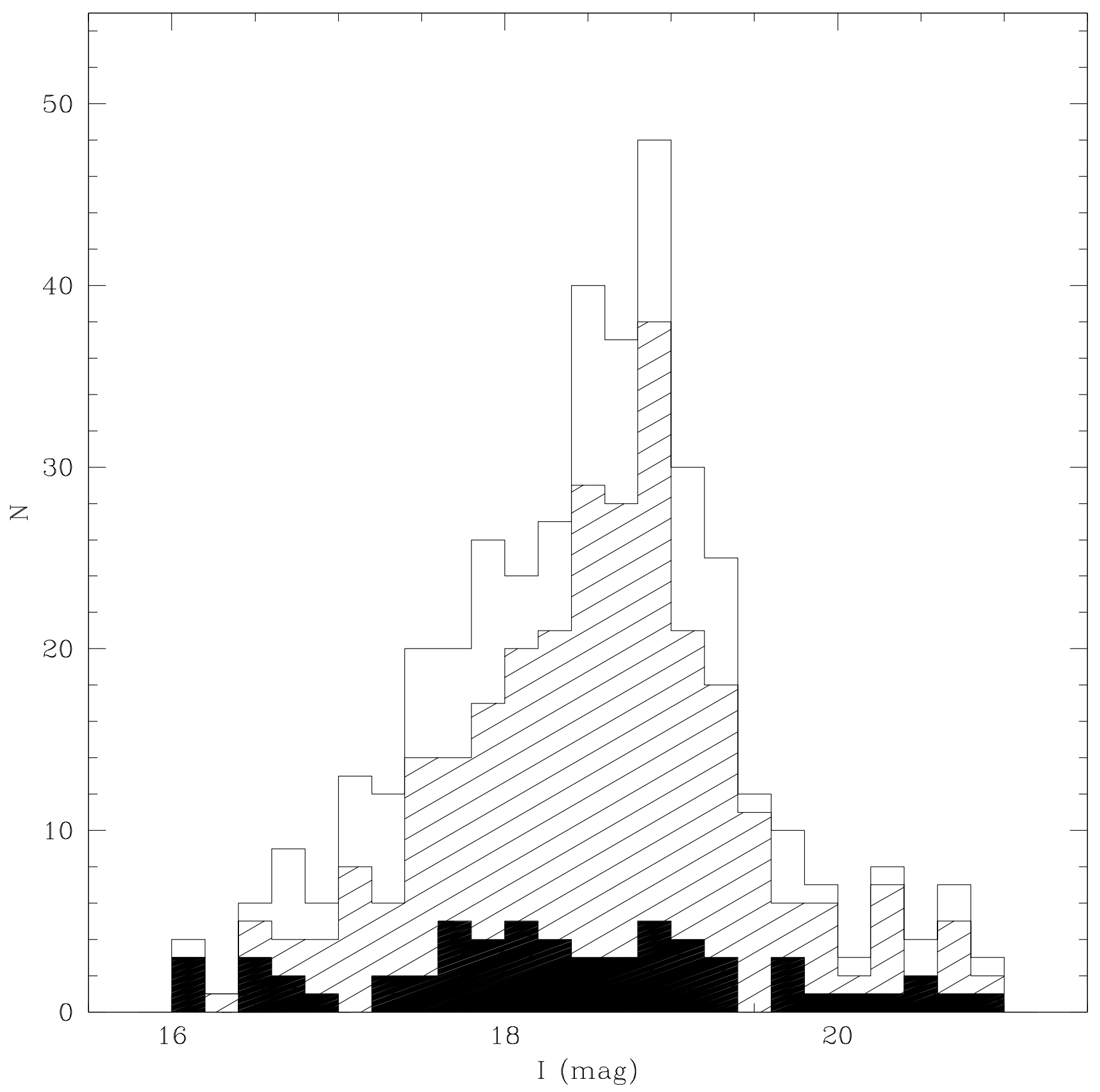




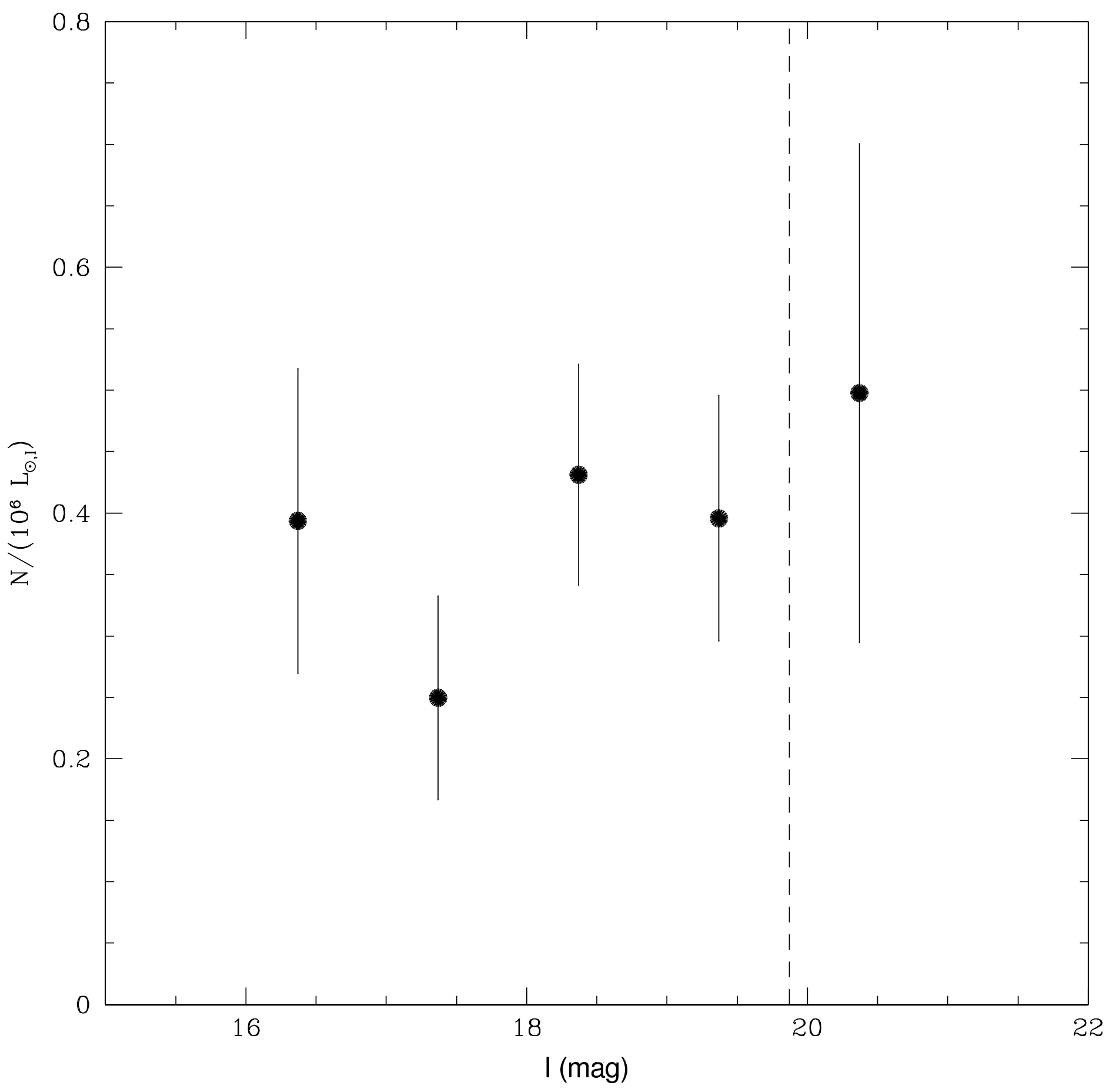

